\title{
Sensitivity vs. Awareness Curve: A Novel Model-Based Analysis to Uncover the Processes Underlying Nonconscious Perception
}

\author{
Ali Pournaghdali, Bennett L. Schwartz, Jason Hays, and Fabian A. Soto \\ Florida International University \\ Department of Psychology
}

\begin{abstract}
In this study, we present a novel model-based analysis of the association between awareness and perceptual processing based on a multidimensional version of signal detection theory (general recognition theory, or GRT). The analysis fits a GRT model to behavioral data and uses the estimated model to construct a sensitivity vs. awareness ( $\mathrm{SvA}$ ) curve, representing sensitivity in the discrimination task at each value of relative likelihood of awareness. This approach treats awareness as a continuum rather than a dichotomy, but also provides an objective benchmark for low likelihood of awareness. In two experiments, we assessed nonconscious facial expression recognition using SvA curves in a condition in which faces (fearful vs. neutral) were rendered invisible using continuous flash suppression (CFS) for 500 and 700 milliseconds. We predicted and found nonconscious processing of face emotion, in the form of higher than chance-level sensitivity in the area of low likelihood of awareness.
\end{abstract}

Keywords: Nonconscious perception; signal detection theory; General recognition theory; Continuous flash suppression; Sensitivity vs. awareness curve

Critical to the dissociation between conscious and nonconscious perception is the assessment of consciousness (or awareness). We evaluate awareness using either subjective or objective measures (Persuh, 2018). Subjective measures are those in which participants provide a report of their subjective experiences (Dehaene and Naccache, 2001; Lau, 2008). Objective measures are those in which performance in a discrimination task is considered as a proxy for conscious perception (Eriksen, 1960). An advantage of subjective measures is their face validity, as they assess the subjective nature of conscious perception directly (Lau, 2008). Objective measures, on the other hand, are measures of performance which may not require consciousness (Lau, 2008), and thus they have received criticism in the literature.

Although subjective measures appear better at evaluating conscious perception, methodological concerns regarding these measures need to be addressed. First, a common practice is to contrast performance in a perceptual discrimination task (e.g. $m$-alternative forced choice, adaptation or priming task) against chance level only in the unaware trials (e.g., Anderson et al., 2012; Koenig and Ro, 2019; Zabelina et al., 2013). Unfortunately, this approach is flawed, as the post-hoc analysis of supposedly unaware trials may lead to above-chance discrimination performance in the perceptual

We would like to thank Dr. Julien Dubois for providing us with the Mondrian images, and Dr. Aaron T. Mattfeld for his comments on an earlier version of this manuscript. discrimination task simply because of the statistical artifact, regression to the mean ${ }^{1}$ (Shanks, 2017; also see Schmidt, 2015).

Therefore, any analysis should incorporate data from aware and unaware trials. Moreover, in any subjective task, a participant uses a relatively arbitrary criterion to classify their perceptual experiences as aware and unaware (Schmidt, 2015). More importantly, participants tend to adopt a conservative criterion in a subjective task in comparison to a perceptual discrimination task (Cowey, 2010; Goldiamond, 1958 ) and this could explain some of the observed nonconscious effects in previous studies (see Heeks and Azzopardi, 2015).

One possible solution to these problems is to use signal detection theory (see Green and Swets, 1966; Macmillan and Creelman, 2005) to analyze awareness and perceptual dis-

\footnotetext{
${ }^{1}$ When we categorize trials as aware and unaware and estimate participants' perceptual discrimination performance in each category, we may overestimate the mean discrimination accuracy in the unaware trials. This happens because of the phenomenon of regression to the mean. That is, with an imperfect correlation between the awareness and performance measures, post-hoc selection of trials based on extreme values of awareness (i.e., trials classified as "unaware") will necessarily lead to a less extreme mean value for performance in those trials (i.e., closer to the mean of all trials). This is a critical but neglected statistical artifact that may influence the results of studies of nonconscious perception. A comprehensive discussion of this phenomenon is beyond the scope of this study. Hence, we refer readers to other publications that investigated this phenomenon more in depth (e.g., Shanks, 2017).
} 
crimination. In SDT, a measure of interest is sensitivity $\left(d^{\prime}\right)$, which serves as the index for the discriminability of signal from noise. To use SDT for studying nonconscious perception, the sensitivities of the awareness and perceptual discrimination tasks are compared, and a difference is considered as a sign of dissociation between conscious and nonconscious perception (e.g., Azzopardi and Cowey, 1997; Heeks and Azzopardi, 2015; Yoshida and Isa, 2015). Note that we can compare two sensitivity measures only if both the awareness and perceptual discrimination tasks measure the same underlying construct (e.g. stimulus detection) using two different formats (e.g. yes/no and 2-interval forced choice tasks; see Heeks and Azzopardi, 2015). A more interesting scenario is one in which two different tasks are analyzed: a subjective awareness report and a task involving discrimination of some stimulus feature (e.g., shape; see Lau and Passingham, 2006). In this case, one approach is to find cases in which some factor affects sensitivity in the subjective awareness task while keeping discrimination performance intact (Lloyd et al., 2013; Maniscalco et al., 2016). Whereas studies using this approach show that there is a dissociation between conscious and nonconscious perception, they do not answer some of the most interesting questions in the field: Is perceptual processing ${ }^{2}$ dependent on awareness ${ }^{3}$, and can it happen in the absence of awareness?

We can formalize and answer such questions using a multidimensional version of SDT, called general recognition theory (GRT; Ashby and Soto, 2015; Ashby and Townsend, 1986; Soto et al., 2017). Here, we present a novel modelbased analysis of data within the framework of GRT, for experiments gathering subjective reports of awareness and perceptual discrimination within each trial.

\section{Sensitivity vs. awareness ( $\mathrm{SvA}$ ) curves: A novel model- based analysis of the interaction between awareness and perceptual processing.}

To use GRT in the study of conscious and nonconscious perception, participants are required to perform two tasks: a subjective awareness task with at least two levels ("stimulus present" and "stimulus absent" or catch trials) and a perceptual discrimination between at least two levels of a perceptual dimension (e.g., in our study, fearful vs. neutral facial expression). GRT describes the perceptual representations in such tasks in a two-dimensional space (Figure 1a): stimulus value along the dimension of interest (e.g., facial expression) in the $x$-axis and awareness of the stimulus in the $y$-axis (mathematical details of the model and other aspects of the analysis are described in detail in the Supplementary Material). Each stimulus is represented by a two-dimensional normal distribution, represented by an ellipse in Figure 1a. The catch trials are represented by a "noise only" distribution, in blue, centered at zero in both dimensions. The two stimuli are represented by distributions higher in awareness and away from zero in opposite directions along the $x$-axis, corresponding to opposite expressions (neutral in red and fearful in teal).

Once this model is estimated from data, it is possible to use it to obtain an SvA curve. For a fixed value of awareness (dotted line in Figure 1a), one obtains two values: the ratio between the likelihood of a catch trial (no stimulus to be aware of) and a stimulus trial (a stimulus to be aware of), and the conditional distributions of neutral and fearful faces at that level of awareness, which allow one to compute a sensitivity value. These two values are placed in the $x$ and $y$ axes of the SvA curve, respectively (Figure 1b).

Note that from an SDT standpoint, awareness is a graded variable. Classification into "aware" and "unaware" trials is based on an arbitrary criterion placed by each participant in this graded dimension. Ideally, we would like to have a more "objective" threshold at which we can classify a percept as having such low awareness as being indistinguishable from the absence of a stimulus. Such an "objective" benchmark is provided by the criterion that would be shown by an ideal observer trying to determine whether they should be aware of a stimulus. This criterion is the point at which the likelihood of a catch trial is the same as the likelihood of a stimulus trial, indicated in orange font in Figure 1a-b. When catch trials are included in the design, there is an objective benchmark of what stimuli the observer should not be aware of, as they land in a perceptual area in which "no stimulus" is the most likely event. When this bound is translated to the SvA curve (orange line, Figure 1b), it divides the $x$-axis into regions of high and low likelihood of awareness.

The pattern shown by the SvA curve allows one to answer questions about the perceptual mechanisms underlying responding in the discrimination task (Figure 1b). In particular, one can determine whether perceptual processing necessary for the discrimination task is dependent (changing sensitivity as a function of awareness) or independent (constant sensitivity values, that is, the green circles in Figure 1b) from awareness. If sensitivity drops with awareness, it may fall to zero before reaching the region of low likelihood of awareness (cyan triangles in Figure 1b), resulting in conscious processing only, or after reaching such region (purple crosses in Figure 1b), resulting in conscious and nonconscious processing. An interesting pattern of results for some areas of

\footnotetext{
${ }^{2}$ By perceptual processing we mean processes that extract perceptual information useful for perceptual decision making, with or without an accompanying subjective experience. While awareness may itself be considered perceptual processing, it is characterized by a subjective experience.

${ }^{3}$ Within the framework of multidimensional SDT, a dissociation implies that awareness and perceptual processing are two different dimensions that can be scaled separately, but it says nothing about whether or not stimuli varying along those dimensions are perceptually independent or interactive.
} 


\section{a. GRT Model}

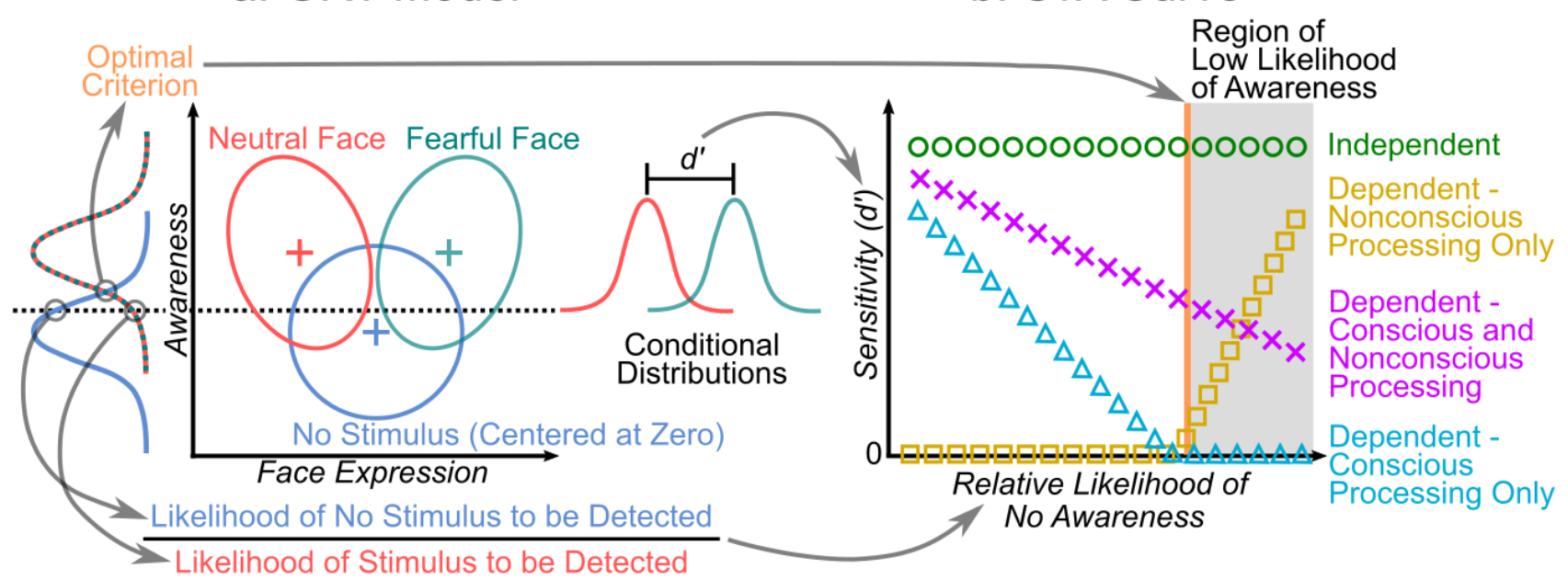

Figure 1. Schematic representation of a model-based analysis of data from an experiment studying the interaction between awareness and perceptual processing. (a) The data is first fitted to a two-dimensional general recognition theory (GRT) model, with one of the dimensions being awareness and the other being a discriminated stimulus value (e.g., facial expression). Each stimulus in this model is represented by a two-dimensional normal distribution (ellipses in the Figure) (b) The estimated model is then used to generate a sensitivity vs. awareness (SvA) curve. For each value in the awareness dimension, two measures are computed: the relative likelihood of no awareness (likelihood of no stimulus to be detected over likelihood of stimulus to be detected) and the conditional sensitivity to the discriminated stimulus value (i.e., conditional $d^{\prime}$ ). An objective bound separating regions with high versus low likelihood of awareness is provided by the criterion of an optimal observer. Different forms of interaction between awareness and perceptual processing produce different patterns in the SvA curve, as shown by the curves with different colors and shapes.

research (see Newell and Shanks, 2014) is the case in which only nonconscious processing is observed (yellow squares). This situation indicates that processing of information might actually improve in the absence of awareness.

\section{The current study}

In two experiments, we rendered fearful and neutral faces invisible for 500 and $700 \mathrm{~ms}$ using continuous flash suppression (CFS; Tsuchiya and Koch, 2005) and assessed nonconscious facial expression recognition using two different SvA curves (Figure 1b): an SvA curve for the discrimination task and an SvA curve for metacognitive confidence judgments. We predicted that we would find evidence for nonconscious facial expression recognition with CFS. That is, we expected to find higher than chance-level sensitivity in the area of low likelihood of awareness for the discrimination and metacognitive judgements. The results corroborated that prediction.

\section{Experiment 1}

The purpose of the first experiment was to evaluate nonconscious facial expression recognition while rendering faces invisible for $500 \mathrm{~ms}$ with CFS.

\section{Methods}

Participants. Sixteen right-handed participants took part in this experiment (11 females, ages 18-24). We recruited the participants from a pool of psychology undergraduate students through Florida International University's (FIU) Psychology Research Participants System (SONA) for one credit/hour compensation. All of the participants had normal or corrected-to-normal (with contact lenses) vision. We did not recruit participants with corrected-to-normal vision using eyeglasses, because of the limitations in our setup. This study was conducted in accordance with the Declaration of Helsinki. The Institutional Review Board of Florida International University approved this study, and written informed consent was obtained from all participants. It is worth noting that model-based analyses are not amenable to traditional power analyses, but simulations with GRT (e.g., Soto et al., 2021) and our previous experience suggest that sample sizes of 10-20 participants per group is sufficient for accurate parameter recovery. Therefore, in both experiments we recruited the number of participants in the middle of this range.

Stimuli. We used a total of 1000 Mondrians. Mondrians are high-contrast, contour-rich images with different patterns of color and shape -named after the artist, Piet Mondrian, because of their resemblance to his work. We used Mondri- 


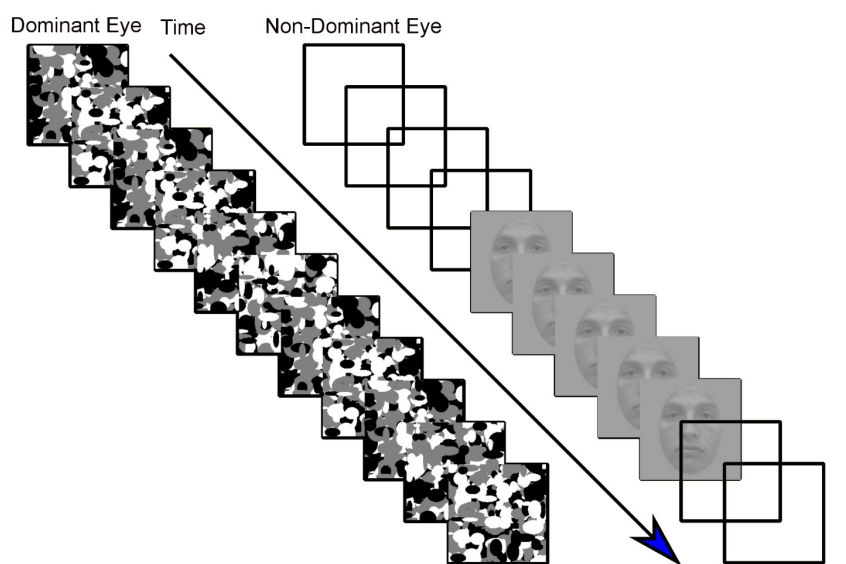

Figure 2. Schematic representation of a single experimental trial.

ans with white, black, and gray filled ovals $(200 \times 200$ pixels $)$ taken (with permission) from the study of Izatt et al. (2014). We chose these Mondrians to keep the masks as similar to the targets as possible and to increase the possibility of suppression (Yang et al., 2014). The targets consisted of faces (neutral and fearful faces, presented in gray scale) of four different identities (two male and two female), extracted from the California Facial Expressions (CAFE) Database (Dailey et al., 2001). To adjust the contrast of the target stimuli, we used GNU Image Manipulation Program (GIMP) version 2.8.18. We reduced the contrast by 31.5 percent (40 out of 127 degrees in GIMP contrast setting), but we kept the images' original brightness. Because the original CAFE images are bigger than the masks, we used GIMP to adjust the size of the target stimuli, so that the masks and the targets would be the same size $(200 \times 200$ pixels $)$. We also presented the faces inside a gray oval to eliminate other facial and bodily parts (like hair and ears) from the image (see Figure 2).

Apparatus. We controlled the stimuli using PsychoPy v1.82.01 (Peirce, 2007) and presented them with an iMAC pro connected to a DELL E773c CRT monitor (17 inch, 1024 $\times 768$ resolution, $60 \mathrm{~Hz}$ refresh rate). Participants viewed the CRT monitor from a distance of about $52 \mathrm{~cm}$ using a set of prism goggles. The goggles are able to induce two separate visual fields: one for each eye. This induces a visual illusion such that the two images (mask and face) appear in the same physical location in the visual field. We also used a screen divider (to ensure that each eye received information only from its own visual field) and a chin rest (to limit participants' head movements).

Procedure. Each trial started with the binocular presentation of a red fixation point inside a box for $500 \mathrm{~ms}$. Then, 11 masks were presented to the participants' right eye (100 $\mathrm{ms}$ each for a total of $1100 \mathrm{~ms}$ ). The target was presented to the left eye at the same time as the fifth mask and remained on the screen for $500 \mathrm{~ms}$ (Figure 2). In one-third of the trials, we did not present any target stimuli, meaning that only the masks were presented on the screen (catch trials). We did not inform participants about the ratio of stimulus present and catch trials, and they did not know about the ratio of these two types of trials. At the end of each trial, participants answered four questions. The first question asked if participants saw the visual stimulus (detection task), and they responded with "yes" or "no." Then, they rated the confidence of their yes/no responses with the following alternatives: "very confident", "somewhat confident", "a little confident", and "not at all confident." The third question asked participants to guess the emotion of the faces (discrimination task) as either "fearful" or "neutral." They were instructed to guess the answer to this question if they did not think they saw the visual target. Finally, they were asked to rate the confidence of their answer to the discrimination question.

Each participant completed a single one-hour session during which they completed two blocks of training trials (eight trials each). After the training trials, participants started the experimental trials (4 blocks and 96 trials in each block). In each block, all nine different types of trials (target-present with one of eight face images -4 identity $\times 2$ emotion-and target-absent) were presented randomly. Participants completed as many trials as they could during the remaining time. On average, each participant completed about 310 trials.

Analysis . The model-based data analysis described earlier (see Figure 1) was performed using R v. 3.5.1 extended with the package grtools v. 0.3.1 (Soto et al., 2017). Details on the GRT model fitted to data, the fitting procedure, the procedure used to obtain the SvA curves, and the parametric bootstrap procedure used to obtain $95 \%$ confidence intervals can all be found in the Supplementary Material. In short, a GRT model (GRT with Individual Differences, or GRTwIND; Soto et al., 2015) was fitted to the observed data. To make sure that the specific version of the model used to build an SvA curve offered a good description of the data without overfitting, we fitted sixteen versions of the model and performed model selection using the Akaike information criterion (AIC; Ashby and Soto, 2015; Soto et al., 2017). Figure 3 shows a summary of the models used. Starting with the full model in which all parameters were fitted to data (leftmost model in Figure 3), we sequentially constrained the model by fixing some of its parameters in the following meaningful ways: (1) we fixed all variances to the same value [VAR1], (2) we assumed that decisions about expression were not influenced by level of awareness [DS(Exp)], (3) we assumed that decisions about awareness were not influenced by level of expression [DS(Awr)], and (4) we assumed that the distribution of awareness values for a stimulus did not depend on the level of expression of that stimulus [PS(Awr)]. As shown in Figure 3, these four individual constraints and combinations of two, three, and all four of them generated a total of sixteen models that were compared. The number of param- 
eters $m$ shown in Figure 3 gives some information about the complexity of each model. The AIC takes into consideration such complexity together with fit to data in selecting the best model.

Each of the models shown in Figure 3 was fitted to the data using maximum likelihood estimation. To ensure that the obtained model included the true maximum likelihood parameter estimates, rather than a local minimum, the optimization algorithm was run 100 times, each time starting from a different random configuration of starting parameters, and the model with highest likelihood was kept. This resulted in sixteen models with parameters fitted to data, which were compared using the AIC in order to choose the best model among them. Detailed reports of the results of this model fit and selection procedure are given in Tables $S 1$ through $S 4$.

From the best GRT model, two values were computed at each value of the awareness dimension (in steps of .01; starting from the smallest mean minus 2 , and ending at the highest mean plus 2): (1) the relative likelihood of no awareness (henceforth, RLNA), which is the likelihood that no stimulus was presented over the likelihood that a stimulus was presented, given a value in the awareness dimension; and (2) the conditional sensitivity, which is a $d^{\prime}$ measure computed from the unidimensional normal distributions of neutral and fearful stimuli, conditional on a value of the awareness dimension (see Figure 1a). To obtain $95 \%$ confidence intervals, 1,000 simulated data samples were generated from the estimated GRT model. The model was then fitted again to each data sample, and the SvA curve was obtained as indicated above. This resulted in an empirical distribution function for SvA curves, and simple percentiles from this function are reported as limits for the $95 \%$ confidence interval.

Note that the RLNA can be computed separately from the marginal distribution of awareness values for fearful and neutral faces shown in Figure 1. Thus, we constructed three different SvA curves from the best GRT model, each with a different RLNA (i.e., $x$-axis): one computed from the neutral face distribution, one from the fearful face distribution, and one that was simply the average of the other two. Creating three SvA curves allows us to evaluate the dependence of visual processing from awareness for each stimulus included, as it can be argued that level of awareness would be different depending on stimulus features such as the presence of an emotional expression.

GRT-wIND is a hierarchical model that includes both group parameters, which determine the overall qualitative pattern shown by the SvA curves, as well as individual attention parameters, which reproduce the same qualitative pattern but shifting the curve up or down. Below we report group-level SvA curves, which are a good way to quickly evaluate the qualitative pattern of results: A slope in the curve indicates that sensitivity depends on awareness, and sensitivity above zero at the optimal bound indicates the pres- ence of unaware sensitivity. However, it is important to highlight that group curves do not provide information about effect sizes.

To ensure that the pattern of results observed in the grouplevel SvA curve was not due to an averaging artifact, and to provide more information about individual-level quantitative patterns of results, we performed a control analysis by building individual SvA curves for every participant.

Although the SvA curve by itself should indicate whether discrimination sensitivity is above chance without awareness, we wanted to evaluate to what extent a model without unaware sensitivity would be able to account for the data. To do this, we fitted a full GRT-wIND model (see leftmost model in Figure 3) with a single constrain: it assumed chance-level discrimination sensitivity in the area of low likelihood of awareness. This model was fitted by penalizing above-chance discrimination sensitivity when RLNA was 1 or higher (see Supplementary Material for a detailed description of the procedure). The full GRT-wIND model was chosen for this analysis to ensure model flexibility, allowing us both to find the best-possible fit to the data from a model without unaware sensitivity, and facilitating the fitting process, as in general it was difficult to fit the parameters of such a model without a failure of the fitting procedure. After fitting the penalized model to our behavioral data, we compared it against the models in Figure 3 using AIC.

We were also interested in evaluating participants' metacognitive sensitivity (meta- $d^{\prime}$; Maniscalco and Lau 2012; 2014) using our SvA curve. To do so, participants' confidence ratings on their discrimination responses were collapsed as follow: "very confident", "somewhat confident" and "a little confident" responses were collapsed in one category (Confident) and "not at all confident" was categorized in the second category (Not-Confident). This was done to ensure that there would be enough data in each response category to allow for model fitting. We used confidence ratings only from the emotional expression (discrimination) task; data from the main subjective awareness detection task was used for the analysis of awareness. This makes comparison of the SvA curves in both analyses easier, as they share a common awareness axis and only differ on how sensitivity is computed.

The analysis of confidence ratings proceeded exactly as the analysis of data from the main discrimination task ${ }^{4}$ (see

\footnotetext{
${ }^{4}$ We did not fit a model constrained to not have unaware sensitivity to the confidence ratings, for two reasons. First, the analysis of discrimination data provided support for the robustness of our SvA-based conclusions regarding unaware discrimination sensitivity, so the additional test seemed unnecessary. Second, fitting a GRT model by penalizing unaware sensitivity proved to be extremely hard, and we anticipated that the issues that we encountered optimizing a penalized likelihood function would only get worse with the more complex GRT model for confidence data.
} 


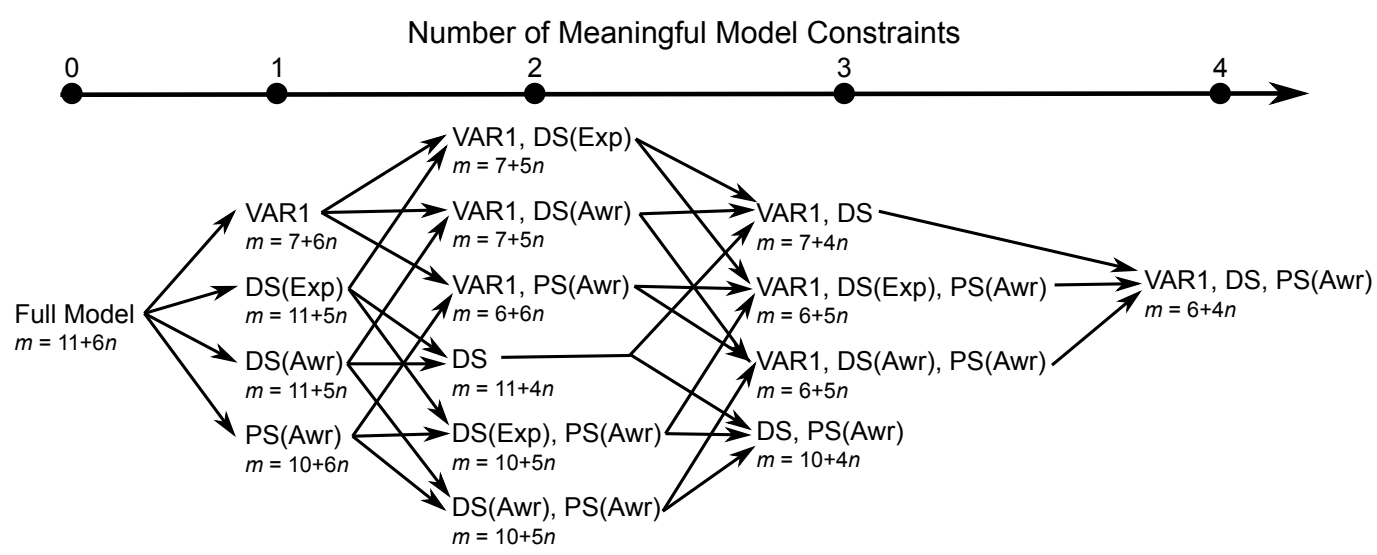

Figure 3. This graph represents the models compared in our analysis, together with their hierarchical relations. At the top of the graph, the rightward arrow represents the direction of our modeling steps from fitting a model with no assumption or constraint to the model with all of the four assumptions. The digits on the arrow represent the number of assumptions assumed for the model in each step. For example, models below digit "2" assume 2 of the 4 assumptions. All the models constructed in this study are depicted below the rightward arrow. The assumptions are shown with the following abbreviations: equal variances [VAR1], decision separability for emotional expression [DS(EXP)], decision separability for awareness [DS(Awr)], perceptual separability for awareness [PS(Awr)]. DS with no specification represents a model that assumes "decision separability for both emotional expression and awareness". The number of parameters $(m)$ is indexed below each model that included number of group and individual parameters. It is important to note that each model results from adding a constraint to a model in the previous step.

Table $S 2$ for the results of model selection). The only difference was that the GRT model fitted to data included two response criteria for the "face expression" dimension, one of them separating "confident neutral" responses from "not-confident" responses, and the other separating "notconfident" responses from "confident fearful" responses. The SvA curve resulting from analysis of confidence rating is theoretically related to the meta- $d^{\prime}$ measure proposed by Maniscalco and Lau $(2012 ; 2014)$. As in meta- $d^{\prime}$, the conditional meta- $d^{\prime}$ in our SvA curve is obtained by determining what $d^{\prime}$ could explain the confidence data under the same distributional assumptions used to compute $d^{\prime}$ from discrimination data. As before, to ensure that the pattern of the results in this SvA curve was not due to an averaging artifact, we constructed individual SvA curves for every participant.

\section{Results}

On average, participants could discriminate face expression in $57.78 \%$ of the total trials and $56.99 \%$ of the unaware trials (the trials that they reported not seeing the target). Moreover, on average participants reported being confident on their discrimination responses in $31.8 \%$ of the trials.

Across both tasks, the model assuming equal variances and decisional separability for awareness was found to be the one that represents the data best (see Tables $S 1$ and $S 2$ ).

The main results are depicted in Figure 4. In all of the graphs, the $x$-axis represents the RLNA, and the $y$-axis represents $d^{\prime}$, computed either from the discrimination task (first row) or from confidence rating responses (meta- $d^{\prime}$; second row). SvA curves computed from the fearful face distribution, neutral face distribution, and their average are depicted in the first, second, and third columns, respectively. In each graph, the blue dotted vertical line is the objective criterion of an ideal observer which divides the $x$-axis into a region of high likelihood of awareness to the left and a region of low likelihood of awareness to the right (see Introduction). In addition, the graphs depict the criteria set by each participant (green dotted vertical lines). In the following paragraphs we discuss the main results from the SvA analysis.

Discrimination task. The selected GRT model provided an excellent fit to the data, accounting for $99.49 \%$ of the observed response proportions (for a graphical depiction of the estimated model and its fit to data, see Figure S1). The pattern of the three SvA curves indicates that perceptual processing of emotional expression is dependent on awareness, as sensitivity drops with relative likelihood of no awareness. There is, however, evidence for nonconscious processing of emotional expression: the three SvA curves are well above a $d^{\prime}$ of zero when they cross the optimal criterion (blue) into the region of low likelihood of awareness. The lower bounds on all of the three curves' confidence interval stay above zero until a point in which the RLNA is between 1.5 to 2 (neutral facial expression $\approx 1.5$, fearful facial expression $\approx 2$, average $\approx 1.7$ ). The conclusion that sensitivity was above chance in the region of low likelihood of awareness was confirmed by the analysis involving a model 

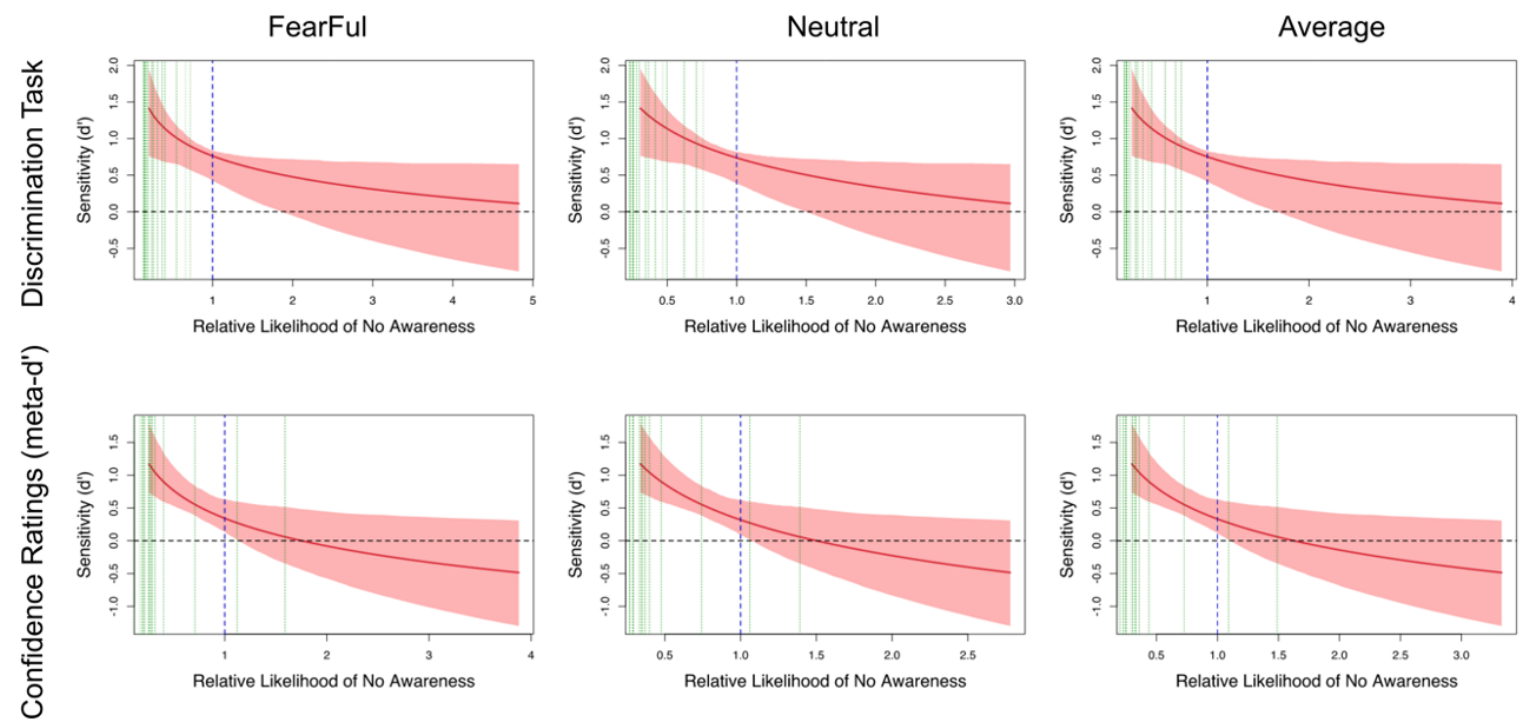

Figure 4. Sensitivity vs. Awareness (SvA) curves obtained from Experiment 1. The top row shows the results of the analyses of the discrimination task and the bottom row shows the results of the analyses of the metacognitive task in which participants were asked to rate their level of confidence on their responses for the main discrimination task. The left column shows the results of the analyses of discrimination (top) and metacognitive (bottom) tasks exclusively for the fearful facial expression. The middle column shows the results of the analyses of discrimination (top) and metacognitive (bottom) tasks exclusively for the neutral facial expression. The right column shows the results of the analyses of discrimination (top) and metacognitive (bottom) tasks for both fearful and neutral facial expressions (average). The solid red line represents the SvA curve obtained from the model, and the lighter red bands represent $95 \%$ confidence intervals. The dotted blue line represents the optimal bound, separating regions of high likelihood of awareness to the left and low likelihood of awareness to the right. The dotted green lines represent the bounds estimated for different participants. The horizontal black dotted line represents zero sensitivity in the discrimination task.

without unaware sensitivity. The AIC for this model (see Table S1) was higher (i.e., worse fit) than for any of the models included in Figure 3, including the best-fitting model.

The analysis of individual SvA curves confirmed that all participants showed above-chance discrimination sensitivity in the region of low likelihood of awareness (see Figure S5). This provided additional support for the group-level SvA results.

As suggested by previous research, the participants' response criteria for the awareness response were rather conservative: they required much more evidence for the presence of a stimulus to respond "aware" than the optimal observer. This can be seen in Figure 4 by comparing the position of the green and blue dotted lines.

Confidence ratings. The selected GRT model provided an excellent fit to the data, accounting for $99.11 \%$ of the observed response proportions (for a graphical depiction of the estimated model and its fit to data, see Figure $S 2$ ). We saw a similar pattern of results as in the analysis of the discrimination task, with no noticeable differences between two types of analysis. The observed pattern of results shows that abovechance metacognitive judgements can be observed even with low likelihood of awareness, or what we have termed nonconscious processing in Figure 1b. The analysis of individual SvA curves not only confirmed this qualitative pattern of results, but showed stronger quantitative evidence of unaware metacognitive sensitivity (see Figure S6).

\section{Experiment 2}

A low number of self-reported "aware" trials in the first experiment might have impacted our model-based analysis, especially because the data used to fit the model might not have enough structure to truly constrain inferences. The presentation duration of a target within the CFS paradigm is known to impact the rate of aware trials (Kaunitz et al., 2014). Hence, in the second experiment we presented the target faces for a longer duration $(700 \mathrm{~ms})$, to obtain more "aware" trials. We expected to see similar results as Experiment 1 .

\section{Methods}

Participants . Thirteen healthy and right-handed participants participated in this experiment (8 females, ages 18- 
41). We recruited the participants from a pool of psychology undergraduate students through the FIU SONA system for one credit/hour compensation. Our recruitment criteria were identical to that of Experiment 1. None of these participants participated in the first experiment. This study was conducted in accordance with the Declaration of Helsinki. The Institutional Review Board of Florida International University approved this study, and written informed consent was obtained from all participants.

Materials and procedure . This experiment was identical to Experiment 1, with two exceptions. First, the target stimulus was presented for $700 \mathrm{~ms}$. Second, we used 13 masks (100 ms each for a total of $1300 \mathrm{~ms}$ ) in each trial to adjust the design to the new target presentation time. Like Experiment 1, we presented the target at the same time with the fifth mask, but it remained on the screen for $700 \mathrm{~ms}$. With these changes, we kept other conditions (such as the number of masks that were presented before and after target presentation) same as in the first experiment. On average, each participant completed about 268 trials.

Analysis . We performed the same model-based analysis as in Experiment 1 (see above).

\section{Results}

On average, participants could discriminate the emotion of the faces in $65.89 \%$ of the total trials and $56.65 \%$ of the unaware trials. Moreover, on average participants reported being confident on their discrimination responses in $57.37 \%$ of the trials.

Across both tasks, the model assuming equal variances and decisional separability for awareness was found to be the one that represents the data best (see Tables S3 and S4). This replicates the results from Experiment 1.

The main results are depicted in Figure 5, which represents the group level SvA curves. In the following paragraphs we discuss the main results from the SvA analysis.

Discrimination task . The results from the discrimination task were similar to the results in Experiment 1. The estimated models provided a good fit to the data, albeit slightly lower than that found from Experiment 1, accounting for $97.93 \%$ of the observed response proportions (for a graphical depiction of the estimated model and its fit to data, see Figure S3). The observation that sensitivity was above chance in the region of low likelihood of awareness (see Figure 5, top row) was confirmed by the analysis involving a model without unaware sensitivity. The AIC for this model (see Table S3) was higher (i.e., worse fit) than for eight other models, including the best-fitting model. All these results were confirmed in the analysis of individual SvA curves (see Figure S7).

Confidence ratings . The estimated model provided a good fit to the data, although again marginally lower than that found from Experiment 1, accounting for $98.72 \%$ of the observed response proportions (for a graphical depiction of the estimated model and its fit to data, see Figure 54 ). Analysis of confidence ratings support the same conclusions as in Experiment 1, and these results were once again obtained at the level of individual SvA curves (see Figure S8), which not only showed the same qualitative pattern of results as the group-level curves, but also much stronger quantitative evidence of unaware meta-cognitive sensitivity.

\section{Discussion}

In two experiments, we found evidence for nonconscious facial expression recognition with CFS using a novel modelbased analysis based on GRT. According to our results, facial expression recognition survives in the absence of awareness, even though its strength depends on the level of awareness: as the RLNA increases, the strength of facial expression recognition drops. This is in accordance with the previous literature on nonconscious facial expression recognition with CFS (e.g., Anderson et al., 2012; Lapate et al., 2016; Yang et al., 2007), even though some low-level features may impact this effect (e.g., Gray et al., 2013; Hedger et al., 2015).

We also found evidence for metacognition sensitivity in the absence of awareness. That is, metacognitive confidence ratings reflected an accurate underlying mechanism of perceptual evaluation, even in the area of low likelihood of awareness. The strength of metacognitive sensitivity, however, depends on the level of awareness. Therefore, metacognitive processes may survive under influence of CFS (for similar results with backward masking, see Jachs et al., 2015). This means that metacognition is dissociable from awareness and may not require awareness in all cases. These results challenge some influential models of awareness and metacognition (e.g., Shimamura, 2008; but see Reder and Schunn, 1996).

Moreover, the similarity between the pattern of results from perceptual discrimination and metacognitive judgements (awareness-dependent and nonconscious perceptual processing), is in line with the SDT assumption that both processes are based on the same input (Galvin et al., 2003; Maniscalco and Lau, 2012) but against results that, while upholding that assumption, have shown that $d^{\prime}$ and meta$d^{\prime}$ are different for the same task (Jachs et al., 2015; Maniscalco and Lau, 2012) and stem from different perceptual evidence (Maniscalco et al., 2016). Although quantitative differences were found in both experiments, the qualitative pattern of results is in agreement across experiments and performance measures. Thanks to our novel model-based approach, this is the first report of nonconscious perceptual processing of facial expression that has controlled for response bias, while providing an objective benchmark to determine nonconscious processing.

Beyond this particular study, our model-based analysis approach is an improvement over previous SDT-based ap- 

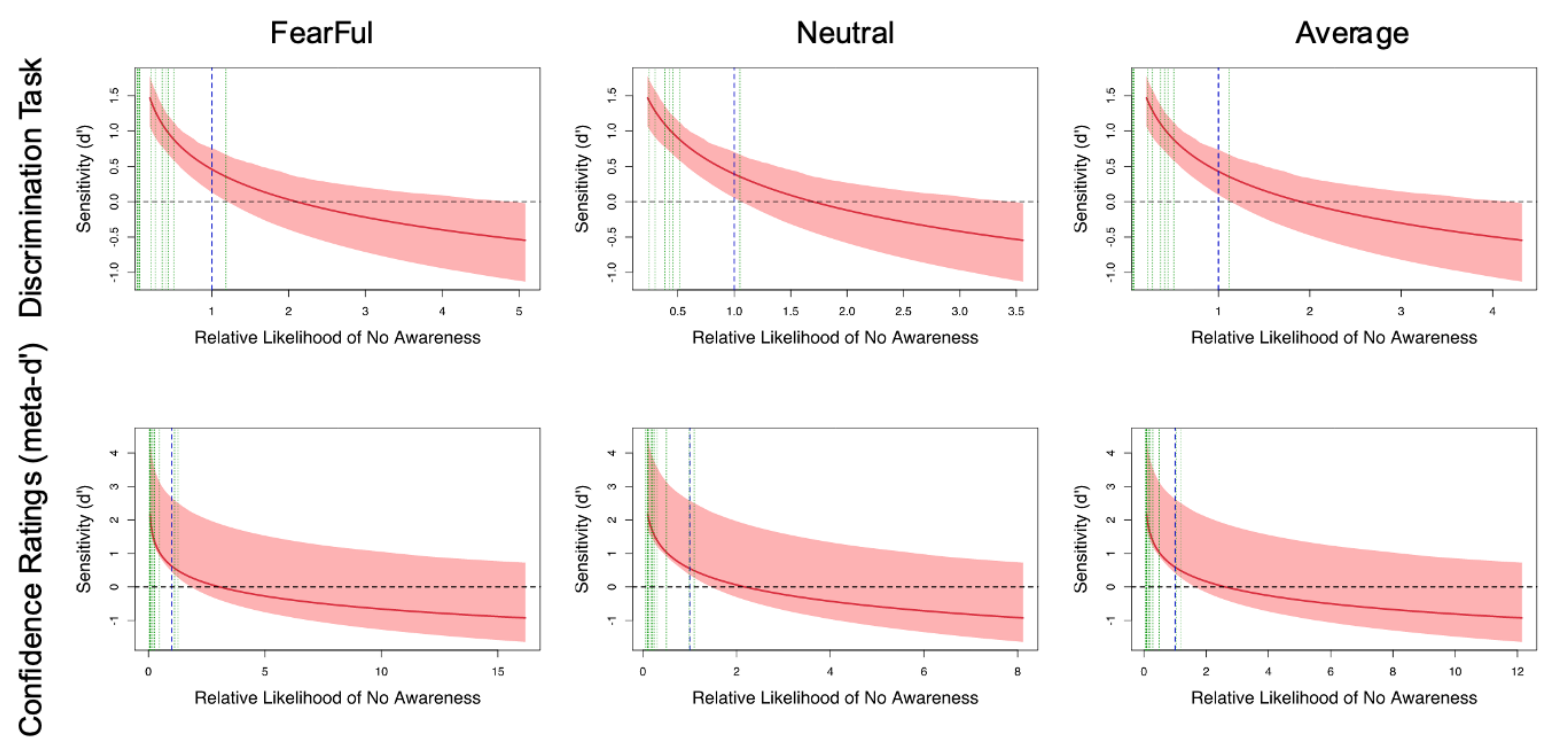

Figure 5. Sensitivity vs. Awareness (SvA) curves obtained from Experiment 2. The top row shows the results of the analyses of the discrimination task and the bottom row shows the results of the analyses of the metacognitive task in which participants were asked to rate their level of confidence on their responses for the main discrimination task. The left column shows the results of the analyses of discrimination (top) and metacognitive (bottom) tasks exclusively for the fearful facial expression. The middle column shows the results of the analyses of discrimination (top) and metacognitive (bottom) tasks exclusively for the neutral facial expression. The right column shows the results of the analyses of discrimination (top) and metacognitive (bottom) tasks for both fearful and neutral facial expressions (average). The solid red line represents the SvA curve obtained from the model, and the lighter red bands represent $95 \%$ confidence intervals. The dotted blue line represents the optimal bound, separating regions of high likelihood of awareness to the left and low likelihood of awareness to the right. The dotted green lines represent the bounds estimated for different participants. The horizontal black dotted line represents zero sensitivity in the discrimination task.

proaches (Azzopardi and Cowey, 1997; Maniscalco and Lau, 2012), as it captures the same psychophysical effects with a higher resolution than previous approaches, allowing us to compare sensitivity across different levels of awareness. Additionally, it can be applied to any design involving a subjective awareness report and a perceptual task, allowing researchers to answer some of the most important questions in the field: Is perceptual processing dependent on awareness, and can it happen in the absence of awareness? We believe that utilizing this approach will help us to more accurately understand the dissociation between conscious and nonconscious perception, and the level of information processing in the absence of awareness across different suppression paradigms and perceptual tasks. As GRT has been extended to the study of neural encoding (Soto et al., 2018), this will eventually help us to gain a better insight about the neuro-computational mechanisms of consciousness.

\section{Open practice statement}

The data for the experiments reported here, as well as $\mathrm{R}$ syntax used to perform the analyses are available at https://osf.io/tnx3w.

\section{References}

Anderson, E., Siegel, E., White, D., and Barrett, L. F. (2012). Out of sight but not out of mind: Unseen affective faces influence evaluations and social impressions. Emotion, 12(6):1210-1221.

Ashby, F. G. and Soto, F. A. (2015). Multidimensional signal detection theory. In Busemeyer, J., Townsend, J. T., Wang, Z. J., and Eidels, A., editors, Oxford Handbook of Computational and Mathematical Psychology, pages 13-34. Oxford University Press, New York, NY.

Ashby, F. G. and Townsend, J. T. (1986). Varieties of perceptual independence. Psychological Review, 93(2):154179.

Azzopardi, P. and Cowey, A. (1997). Is blindsight like normal, near-threshold vision? Proceedings of the $\mathrm{Na}$ tional Academy of Sciences, 94(25):14190-14194.

Cowey, A. (2010). The blindsight saga. Experimental Brain Research, 200(1):3-24.

Dailey, M., Cottrell, G. W., and Reilly, J. (2001). California facial expressions, CAFE. 
Dehaene, S. and Naccache, L. (2001). Towards a cognitive neuroscience of consciousness: basic evidence and a workspace framework. Cognition, 79(1):1-37.

Eriksen, C. W. (1960). Discrimination and learning without awareness: A methodological survey and evaluation. Psychological Review, 67(5):279-300.

Galvin, S. J., Podd, J. V., Drga, V., and Whitmore, J. (2003). Type 2 tasks in the theory of signal detectability: Discrimination between correct and incorrect decisions. Psychonomic Bulletin $\mathcal{E}$ Review, 10(4):843-876.

Goldiamond, I. (1958). Indicators of perception: I. Subliminal perception, subception, unconscious perception: An analysis in terms of psychophysical indicator methodology. Psychological Bulletin, 55(6):373-411.

Gray, K. L. H., Adams, W. J., Hedger, N., Newton, K. E., and Garner, M. (2013). Faces and awareness: Lowlevel, not emotional factors determine perceptual dominance. Emotion, 13(3):537-544.

Green, D. M. and Swets, J. A. (1966). Signal detection theory and psychophysics. Wiley, New York.

Hedger, N., Adams, W. J., and Garner, M. (2015). Fearful faces have a sensory advantage in the competition for awareness. Journal of Experimental Psychology: Human Perception and Performance, 41(6):1748-1757.

Heeks, F. and Azzopardi, P. (2015). Thresholds for detection and awareness of masked facial stimuli. Consciousness and Cognition, 32:68-78.

Izatt, G., Dubois, J., Faivre, N., and Koch, C. (2014). A direct comparison of unconscious face processing under masking and interocular suppression. Frontiers in Psychology, 5:659.

Jachs, B., Blanco, M. J., Grantham-Hill, S., and Soto, D. (2015). On the independence of visual awareness and metacognition: A signal detection theoretic analysis. Journal of Experimental Psychology: Human Perception and Performance, 41(2):269-276.

Kaunitz, L. N., Fracasso, A., Skujevskis, M., and Melcher, D. (2014). Waves of visibility: probing the depth of inter-ocular suppression with transient and sustained targets. Frontiers in Psychology, 5:804.

Koenig, L. and Ro, T. (2019). Dissociations of conscious and unconscious perception in TMS-induced blindsight. Neuropsychologia, 128:215-222.

Lapate, R. C., Rokers, B., Tromp, D. P. M., Orfali, N. S., Oler, J. A., Doran, S. T., Adluru, N., Alexander, A. L., and Davidson, R. J. (2016). Awareness of Emotional Stimuli Determines the Behavioral Consequences of Amygdala Activation and Amygdala-Prefrontal Connectivity. Scientific Reports, 6:25826.

Lau, H. (2008). Are we studying consciousness yet? In Weiskranz, L. and Davies, M., editors, Frontiers of Consciousness: Chichele Lectures, volume 2008, pages 245-258. Oxford University Press, New York,
NY.

Lau, H. and Passingham, R. E. (2006). Relative blindsight in normal observers and the neural correlate of visual consciousness. Proceedings of the National Academy of Sciences, 103(49):18763-18768.

Lloyd, D. A., Abrahamyan, A., and Harris, J. A. (2013). Brain-stimulation induced blindsight: Unconscious vision or response bias? PLOS ONE, 8(12):e82828.

Macmillan, N. A. and Creelman, C. D. (2005). Detection theory: A user's guide. Lawrence Erlbaum Associates, Mahwah, NJ, 2nd edition.

Maniscalco, B. and Lau, H. (2012). A signal detection theoretic approach for estimating metacognitive sensitivity from confidence ratings. Consciousness and Cognition, 21(1):422-430.

Maniscalco, B. and Lau, H. (2014). Signal detection theory analysis of type 1 and type 2 data: Meta-d', responsespecific meta-d', and the unequal variance SDT model. In Fleming, S. M. and Frith, C. D., editors, The Cognitive Neuroscience of Metacognition, pages 25-66. Springer Berlin Heidelberg, Berlin, Heidelberg.

Maniscalco, B., Peters, M. A. K., and Lau, H. (2016). Heuristic use of perceptual evidence leads to dissociation between performance and metacognitive sensitivity. Attention, Perception, $\mathcal{E}$ Psychophysics, 78(3):923-937.

Newell, B. R. and Shanks, D. R. (2014). Unconscious influences on decision making: A critical review. Behavioral and Brain Sciences, 37(1):1-19.

Peirce, J. W. (2007). PsychoPy-Psychophysics software in Python. Journal of Neuroscience Methods, 162(1-2):8-13.

Persuh, M. (2018). Measuring perceptual consciousness. Frontiers in Psychology, 8:2320.

Reder, L. M. and Schunn, C. D. (1996). Metacognition does not imply awareness: Strategy choice is governed by implicit learning and memory. In Reder, L. M., editor, Implicit memory and metacognition, pages 45-78. Psychology Press, New York, NY.

Schmidt, T. (2015). Invisible stimuli, implicit thresholds: Why invisibility judgments cannot be interpreted in isolation. Advances in Cognitive Psychology, 11(2):31-41.

Shanks, D. R. (2017). Regressive research: The pitfalls of post hoc data selection in the study of unconscious mental processes. Psychonomic Bulletin $\mathcal{E}$ Review, 24(3):752-775.

Shimamura, A. P. (2008). A neurocognitive approach to metacognitive monitoring and control. In Dunlosky, J. and Bjork, R. A., editors, Handbook of metamemory and memory., pages 373-390. Psychology Press, New York, NY, US.

Soto, F. A., Stewart, R. A., Hosseini, S., Hays, J. S., 
and Beevers, C. G. (2021). A computational account of the mechanisms underlying face perception biases in depression. Journal of Abnormal Psychology, 130(5):443-454.

Soto, F. A., Vucovich, L., Musgrave, R., and Ashby, F. G. (2015). General recognition theory with individual differences: A new method for examining perceptual and decisional interactions with an application to face perception. Psychonomic Bulletin $\mathcal{F}$ Review, 22(1):88111.

Soto, F. A., Vucovich, L. E., and Ashby, F. G. (2018). Linking signal detection theory and encoding models to reveal independent neural representations from neuroimaging data. PLOS Computational Biology, 14(10):e1006470.

Soto, F. A., Zheng, E., Fonseca, J., and Ashby, F. G. (2017). Testing separability and independence of perceptual dimensions with general recognition theory: a tutorial and new R package (grtools). Frontiers in Psychology,
$8: 696$.

Tsuchiya, N. and Koch, C. (2005). Continuous flash suppression reduces negative afterimages. Nature Neuroscience, 8(8):1096.

Yang, E., Brascamp, J., Kang, M. S., and Blake, R. (2014). On the use of continuous flash suppression for the study of visual processing outside of awareness. Frontiers in Psychology, 5:724.

Yang, E., Zald, D. H., and Blake, R. (2007). Fearful expressions gain preferential access to awareness during continuous flash suppression. Emotion, 7(4):882-886.

Yoshida, M. and Isa, T. (2015). Signal detection analysis of blindsight in monkeys. Scientific Reports, 5:10755.

Zabelina, D. L., Guzman-Martinez, E., Ortega, L., Grabowecky, M., Suzuki, S., and Beeman, M. (2013). Suppressed semantic information accelerates analytic problem solving. Psychonomic Bulletin $\mathcal{E}$ Review, 20(3):581-585. 


\section{Supplementary Material}

Sensitivity vs. Awareness Curve: A Novel Model-Based Analysis

to Uncover the Processes Underlying Nonconscious Perception

Ali Pournaghdali, Bennett L. Schwartz, Jason Hays, and Fabian A. Soto

\section{Contents}

I General recognition theory analysis of perceptual awareness and sensitivity (GRTapas)

1 GRT-wIND model description $\quad 1$

2 Model Selection Procedure $\quad 3$

3 Interaction analysis $\quad 3$

3.1 Conditional sensitivity . . . . . . . . . . . . . . . . . . . . . . . . 3

3.2 Relative likelihood of no awareness . . . . . . . . . . . . . . . . . . . . . . . 4

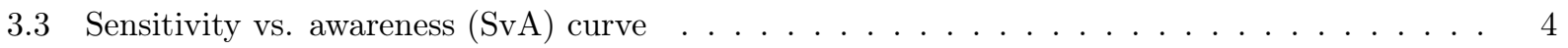

4 How to obtain an SvA curve using grtools 5

II Supplementary results $\quad 6$

5 Model Comparison and Selection $\quad 7$

5.1 Model Penalizing Unaware Sensitivity . . . . . . . . . . . . . . . . . . . 7

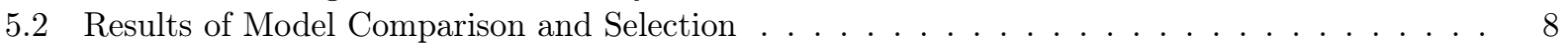

6 Estimated GRT-wIND models $\quad 10$

$\begin{array}{llr}7 & \text { Individual SvA Curves } & 12\end{array}$

$\begin{array}{ll}\text { References } & 15\end{array}$

Part I

General recognition theory analysis of perceptual awareness and sensitivity (GRTapas)

\section{GRT-wIND model description}

The model fitted to data from our continuous flash suppression experiments is a special case of GRT-wIND (Soto et al., 2015). This is a multi-level model, with "group parameters" representing aspects of perceptual 
processing considered to be common to all participants in an experiment, and "individual parameters" representing aspects of perceptual processing and decision making considered to vary across individuals. This model offers several advantages over traditional individual-level GRT models: it is more parsimonious, avoids over-fitting, avoids parameter identifiability problems with such models, and fits experimental data better (see Soto et al., 2015).

The task involved two stimuli (neutral and fearful face stimuli) and "catch" trials in which no stimulus was presented (noise stimulus). These three stimulus conditions, indexed by $s$, were each represented by a bivariate normal distribution described by a mean vector:

$$
\mu_{s}=\left[\begin{array}{l}
\mu_{s, x} \\
\mu_{s, y}
\end{array}\right]
$$

and a covariance matrix:

$$
\boldsymbol{\Sigma}_{s}=\left[\begin{array}{cc}
\sigma_{s, x}^{2} & \rho_{s} \sigma_{s, x} \sigma_{s, y} \\
\rho_{s} \sigma_{s, x} \sigma_{s, y} & \sigma_{s, y}^{2}
\end{array}\right]
$$

where $x$ is the first dimension of the model, representing perception of emotional expression, $y$ is the second dimension of the model, representing awareness, $\sigma$ is a standard deviation parameter and $\rho$ is a correlation parameter. We set $\mu_{\text {noise }}=[0,0]$ to fix the position of the final solution, and $\sigma_{\text {noise }, x}=\sigma_{\text {noise }, y}=1$ to fix the scale of the final solution. In theory, this is sufficient to fix the scale of the final solution, but note that if model selection is not implemented, then we would recommend fixing all variances to one, based on our experience with the model and concerns regarding the recoverability of variance parameters from GRT-wIND (Silbert \& Thomas, 2017). The three bivariate normal distributions just described together comprise the "group parameters" in GRT-wIND.

The model also includes several individual parameters. Two parameters, $\kappa_{k}$ and $\lambda_{k}$, determine the level of attention that participant $k$ allocates to each dimension. The parameter $\kappa_{k}>0$ represents global attention, with high values of it decreasing all variances in the model, which yields better discrimination in both dimensions. The parameter $\lambda_{k}$ represents selective dimensional attention, and ranges from 0 to 1 . A value of $\lambda_{k}=.5$ represents equal attention to both dimensions, with higher values representing more attention to dimension $x$ and lower values representing more attention to dimension $y$. The covariance matrix for the distribution of perceptual effects of stimulus $s$ in participant $k$ is described by the following equation:

$$
\boldsymbol{\Sigma}_{s k}=\left[\begin{array}{cc}
\frac{\sigma_{s, x}^{2}}{\kappa_{k} \lambda_{k}} & \rho_{s} \frac{\sigma_{s, x} \sigma_{s, y}}{\sqrt{\kappa_{k}^{2} \lambda_{k}\left(1-\lambda_{k}\right)}} \\
\rho_{s} \frac{\sigma_{s, x} \sigma_{s, y}}{\sqrt{\kappa_{k}^{2} \lambda_{k}\left(1-\lambda_{k}\right)}} & \frac{\sigma_{s, y}^{2}}{\kappa_{k}\left(1-\lambda_{k}\right)}
\end{array}\right] .
$$

Another set of individual parameters describe the linear decision bounds (i.e., criteria) that are assumed to be unique to each participant. Each bound can be written as a discriminant function:

$$
h_{d k}(X, Y)=b_{d k, x} X+b_{d k, y} Y+c_{d k},
$$

where $r$ indexes a particular discrimination (e.g., $d=$ neutral/fearful, or $d=$ aware/not-aware), and $X$ and $Y$ represent specific values in the dimensions $x$ and $y$, respectively. One parameter in Equation 4 is always fixed to 1 . The other two parameters control the slope of the decision bound and its position. Decision bounds divide the two-dimensional space of the model into response regions. Assume that $R_{1}, R_{2} \ldots R_{n}$ represent the $n$ possible combinations of responses in an experiment. For example, $R_{1}$ could correspond to the response combination "not aware of a stimulus / face was neutral", $R_{2}$ to the response combination "not aware of a stimulus / face was fearful", $R_{3}$ to the response combination "aware of a stimulus / face was neutral", and $R_{4}$ to the response combination "aware of a stimulus / face was fearful". Then the probability of each response combination given the presentation of stimulus $s$, or $P\left(R_{j} \mid s\right)$, is the area of the distribution representing $s$ that falls within the response region assigned to $R_{j}$.

We estimate the parameters of the GRT-wIND model by maximizing the log-likelihood of the model:

$$
L L=\sum_{k} \sum_{s} \sum_{j} r_{k s j} \log P_{k}\left(R_{j} \mid s\right),
$$


where $r_{k s j}$ represents the frequency with which participant $k$ reported the response combination $R_{j}$ when presented with stimulus $s$, and $P_{k}\left(R_{j} \mid s\right)$ is the model prediction of the probability of such event.

\section{Model Selection Procedure}

To make sure that the specific version of the model used to build an SvA curve offered a good description of the data without overfitting, we fitted sixteen competing versions of the model, illustrated in Figure 3 of the main manuscript. Starting with the full model in which all parameters were fitted to data (leftmost model in Figure 3), we sequentially constrained the model by fixing some of its parameters in the following meaningful ways: (1) we fixed all variances to the same value [VAR1], (2) we assumed that decisions about expression were not influenced by level of awareness [DS(Exp)], (3) we assumed that decisions about awareness were not influenced by level of expression [DS(Awr)], and (4) we assumed that the distribution of awareness values for a stimulus did not depend on the level of expression of that stimulus [PS(Awr)]. These four individual constraints and combinations of two, three, and all four of them generated a total of sixteen models that were compared.

We compared the models using the corrected Akaike information criterion (AIC; see Ashby \& Soto, 2015; Soto et al., 2017):

$$
A I C=-L L+2 m+\frac{2 m(m+1)}{n^{2}-m-1},
$$

where $L L$ is the value from Equation 5 at the best-fitting set of model parameters, $m$ is the number of free parameters in the model, and $n$ is the number of cells in the confusion matrix. The first two terms in this equation correspond to the traditional definition of the AIC and the last term corresponds to the correction factor. Lower values of AIC indicate better models.

From a list of AIC values it is possible to compute AIC weights, which can be interpreted as the probability that a model in the set is the closest to the "true" model that generated the observed data. These weights are computed using the following equation:

$$
w_{i}(A I C)=\frac{\exp \left\{-\frac{1}{2} \Delta_{i}(A I C)\right\}}{\sum_{k=1}^{K} \exp \left\{-\frac{1}{2} \Delta_{k}(A I C)\right\}},
$$

where

$$
\Delta_{i}(A I C)=A I C_{i}-\min A I C .
$$

Here $i$ has been used to index the models included in the comparison set, and min $A I C$ represents the smallest $A I C$ in the set.

\section{$3 \quad$ Interaction analysis}

After estimating a GRT-wIND model as described in the previous section, the obtained parameter values can be used to perform an analysis of the interaction between perceptual processing in the discrimination task (i.e., emotional expression task) and awareness. The main product of this analysis is a sensitivity versus awareness (SvA) curve.

\subsection{Conditional sensitivity}

Estimation of a full GRT-wIND model results in three perceptual distributions: (1) a noise distribution, with means $\mu_{\text {noise }, x}$ and $\mu_{\text {noise }, y}$, both fixed to zero, standard deviations $\sigma_{n o i s e, x}$ and $\sigma_{\text {noise,y } y}$, both fixed to one, and correlation $\rho_{\text {noise }}$ that is estimated; (2) a neutral face distribution, with means $\mu_{\text {neutral }, x}$ and $\mu_{\text {neutral }, y}$, standard deviations $\sigma_{\text {neutral }, x}$ and $\sigma_{\text {neutral }, y}$, and correlation $\rho_{\text {neutral }}$, all of them estimated; and (3) a fearful face distribution, with means means $\mu_{\text {fear }, x}$ and $\mu_{\text {fear }, y}$, standard deviations $\sigma_{\text {fear }, x}$ and $\sigma_{\text {fear }, y}$, and correlation $\rho_{\text {fear }}$, all of them estimated. 
These parameters allow one to obtain the distribution of perceptual evidence in the discrimination task, $x$, conditional on a specific value of awareness, $y=Y$, for each stimulus $s$ (i.e., noise, neutral, and fear), which follows a normal distribution with mean:

$$
\mu_{x \mid y=Y, s}=\mu_{s, x}+\frac{\sigma_{s, x}}{\sigma_{s, y}} \rho_{s}\left(Y-\mu_{s, y}\right),
$$

and variance:

$$
\sigma_{x \mid y=Y, s}^{2}=\left(1-\rho_{s}^{2}\right) \sigma_{s, x}^{2} .
$$

This allows us to compute perceptual value sensitivity conditional on awareness evidence:

$$
d_{x \mid y=Y}^{\prime}=\frac{\mu_{x \mid y=Y, \text { fear }}-\mu_{x \mid y=Y, \text { neutral }}}{\sqrt{\frac{\sigma_{x \mid y=Y, \text { fear }}^{2}+\sigma_{x \mid y=Y, \text { neutral }}^{2}}{2}}} .
$$

Note that this is not a single value, but rather a function of $y$.

\subsection{Relative likelihood of no awareness}

Awareness is not a dichotomy in a signal detection model, but instead it depends on a graded variable $y$. Using the estimated GRT-wIND model, we can compute an index of the relative likelihood of no awareness as a function of $y=Y$.

For each stimulus $s$, one can obtain a marginal distribution of $y$ represented by $g_{s}(y)$, which is a univariate normal with mean $\mu_{s, x}$ and standard deviation $\sigma_{s, x}$.

The condition in which no stimulus is presented is represented by the noise distribution in the GRT model, with marginal $g_{\text {noise }}(y)$. This provides an objective benchmark of the area of $y$ where there should be little evidence of awareness. The two conditions in which a stimulus is presented, represented by the neutral and fear distributions in the GRT model, indicate the area in $y$ where awareness becomes more likely. Their marginal distributions are represented by $g_{s}(y)$ with $s=\{$ neutral, fear $\}$. The ratio between the distributions corresponding to these noise and stimulus conditions provides the relative likelihood of no awareness, or RLNA:

$$
R L N A_{s}(y)=\frac{g_{\text {noise }}(y)}{g_{s}(y)}
$$

As the conditional $d^{\prime}$ in Equation 10, this is not a single value, but a function of $y$. Note also that there are two $R L N A$ values, one for each value of $s$. In addition, in the main manuscript we report SvA curves based on the average of $R L N A_{\text {neutral }}$ and $R L N A_{\text {fear }}$.

The $R L N A_{s}$ is a good choice to quantify level of awareness in the model, for two reasons. First, this measure is easy to interpret: it describes the odds that no stimulus has been presented versus that a stimulus has been presented in a trial. Second, this measure provides an objective boundary between areas of high and low likelihood of awareness, at the value of one. An ideal observer whose task is to indicate whether a stimulus has been presented would respond "yes" for $R L N A<1$ and respond "no" for $R L N A>1$. This is an objective benchmark of where there should be a boundary between high- and low-awareness perceptual processing.

\subsection{Sensitivity vs. awareness (SvA) curve}

We can compute conditional $d^{\prime}$ using equation 10 and relative likelihood of no awareness using equation 11 for a range of values of $y$, and then plot the corresponding values in a sensitivity vs. awareness (SvA) curve. One problem is deciding the range of values of $y$ to plot. Because all variances are fixed to one in the best-fitting models for all our analyses (see Tables 1-4 below), $y$ is in units of the standard normal distribution. We plot from the highest mean +2 to the lowest mean -2 . This ensures that only a small fraction (less than 5\%) of the densities is not covered, without over-extending the range of the plot to areas of the underlying variables unlikely to affect overt psychophysical performance. 
Because the GRT-wIND parameter estimates are noisy, the SvA curve computed from them must be noisy as well. To reach conclusions about the interaction between perceptual processing and awareness, one must obtain some measure of the uncertainty regarding the curve's position. To do this, we computed confidence intervals using a parametric bootstrap procedure (Carpenter \& Bithell, 2000; Good, 2006) and plotted them in the SvA curve. We used a parametric rather than a non-parametric bootstrap procedure because (1) our analysis assumes a model for the data distribution, so in this case the parametric bootstrap is appropriate (Carpenter \& Bithell, 2000), and (2) using the nonparametric bootstrap with small sample sizes such as ours leads to imprecise confidence intervals (Good, 2006).

At each step of the bootstrap, a simulated data set was sampled from the fitted GRT-wIND model, and was used to fit a new model (with the same constraints as the original) to the simulated data. The obtained parameters were used to compute an SvA curve whose values were recorded, and the process started again. A total of 1,000 SvA curves were generated this way, and the resulting empirical distribution of SvA curves was used to obtain $95 \%$ confidence intervals at each point of the $R L N A$ variable using a simple quantile procedure.

The resulting SvA plots (see Figures 4 and 5 in the main manuscript) provide information about the interaction between perceptual processing in the discrimination task and awareness. A flat function would indicate that perceptual processing is independent from awareness. On the other hand, a decreasing or increasing function indicates that perceptual processing depends on awareness. We can use the confidence bands to conclude whether the curve deviates from a flat pattern. If the initial and final confidence intervals do not overlap, then one can confidently conclude that the curve is not flat, and perceptual processing depends or interacts with perceptual awareness.

Similarly, one can use the confidence interval at a value of $R L N A=1$ to make conclusions about whether or not nonconscious perceptual processing occurs. If the confidence interval is at or below zero for all $R L N A \geq 1$, this means that there is no noconscious perceptual processing. On the other hand, if the confidence interval is higher than zero at any $R L N A \geq 1$, this means that there is nonconscious perceptual processing (i.e., perceptual processing that is present during low likelihood of awareness).

Another possibility is that the curve is increasing, with perceptual processing being better under conditions of low awareness. In that case, we would expect a positive curve that is not above zero at $R L N A<1$, but becomes higher than zero at $R L N A \geq 1$, providing evidence for nonconscious perceptual processing without conscious perceptual processing (i.e., processing present only during low awareness, but not present during high awareness).

\section{How to obtain an SvA curve using grtools}

We have extended the $\mathrm{R}$ package grtools (Soto et al., 2017) to make it very easy for any researcher to obtain an SvA curve from results of a behavioral experiment. The experimental design must involve obtaining a subjective report of awareness ("aware" or "not aware) and a discrimination response (e.g., "neutral" vs. "fearful" face) in each trial. In order to obtain SvA curves with meta- $d$ ', a judgement of the confidence on the discrimination response (e.g., "confident" vs. "not confident") must also be obtained.

After the data are obtained, they must be summarized into confusion matrices. Soto et al. (2017) give a tutorial on how such confusion matrices must be prepared to fit GRT-wIND to data. In this specific analysis, an important extra step consists of providing a row of zeros for stimulus $A 2 B 1$ (i.e., the second row in each confusion matrix), corresponding to trials which are impossible in nature: no stimulus is presented, but there is a signal for the perceptual discrimination task (e.g., "fearful").

After obtaining a list of confusion matrices called cmats, the full analysis is performed in only 6 lines of code: 


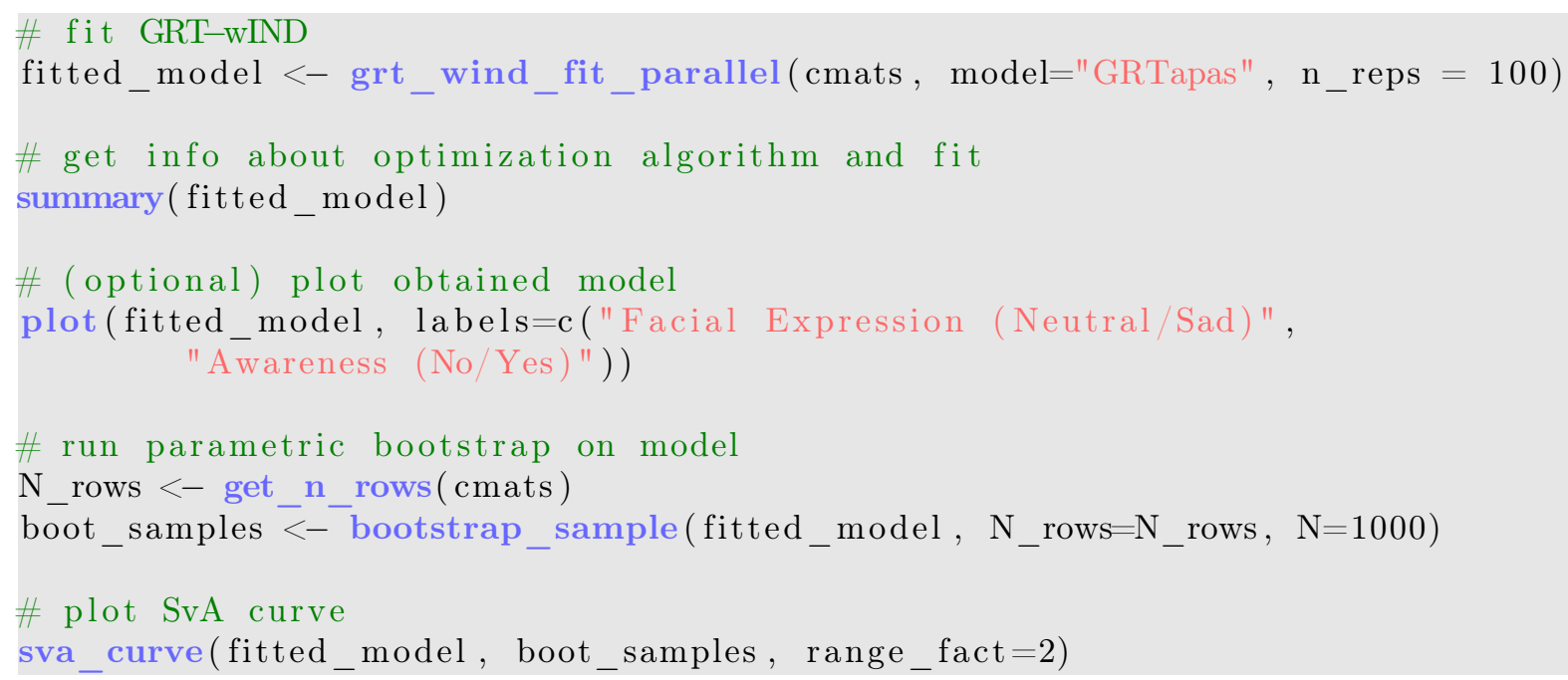

The last line in the previous code produces the average SvA curve. To obtain the SvA curves for specific values of the stimulus (e.g., "neutral" or "fearful"), the only requirement is to include the option stim_level $=\mathrm{c}(\mathrm{S})$, where $\mathrm{S}$ stands for a numerical value (e.g., 1 or 2 ) representing the dimensional level (i.e., A1 or A2; see Ashby \& Soto, 2015; Soto et al., 2017) that one wants to use to obtain the SvA curve.

When multiple GRT-wIND models are fitted to data and compared, the first line of code is repeated multiple times. Constraints in the model can be listed in the model input to the function grt_wind_fit_parallel. For example, let's say that one would want to compare a model in which all variances are fixed to a value of one ("1-VAR" in grtools) against a model in which decisional separability is assumed for awareness $(" \mathrm{DS}(\mathrm{B})$ " in grtools). We can do that with the following code:

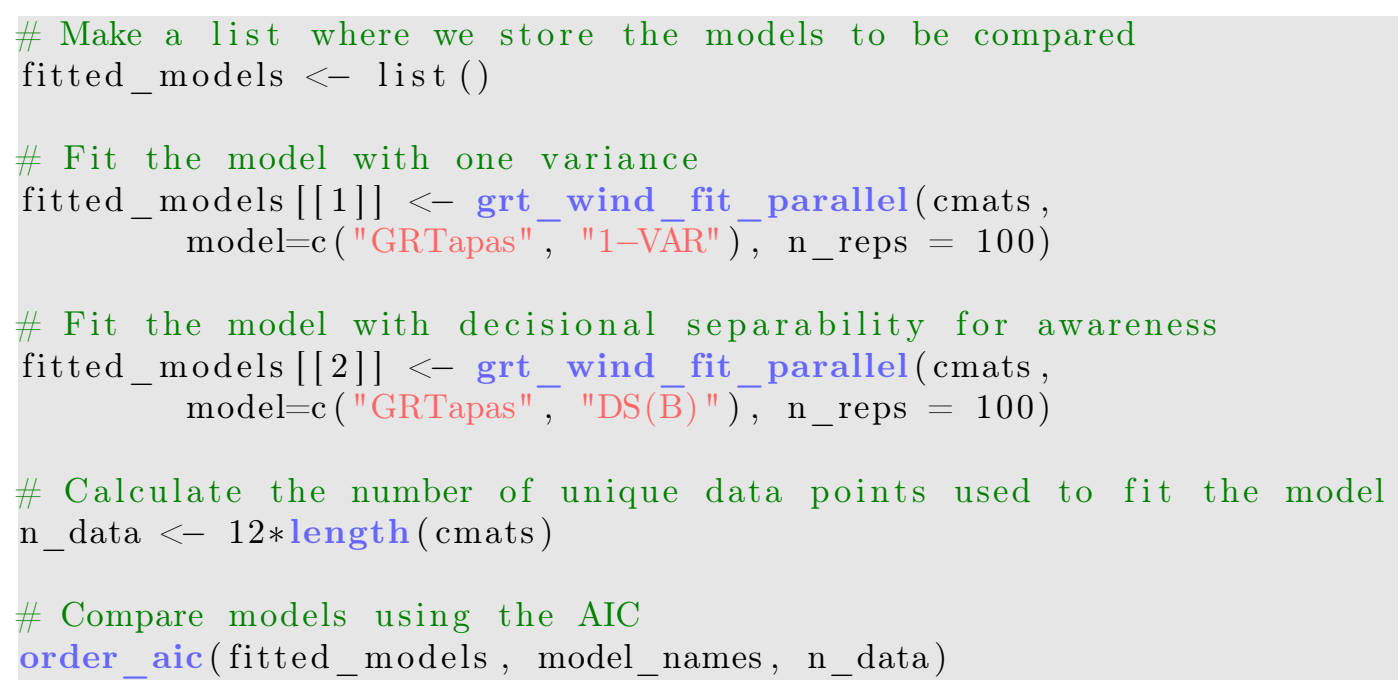

The output of the last line in the previous code is a table like those reported below (Tables 1-4).

Analysis of data from confidence ratings in terms for meta- $d^{\prime}$ is performed in the same way, but including the option model=c("GRTapas","r3x2") within the function grt_wind_fit_parallel. 


\section{Part II \\ Supplementary results}

\section{Model Comparison and Selection}

We fit sixteen different GRT models to the data from each task and in each experiment. Supplementary Tables 1 through 4 summarize the results of model comparison with AIC. Each row in each table represents one model. The first column represents a number assigned to each model in order to identify it (see Figure 3 of the main manuscript; models were numbered from left to right and from top to bottom as they appear in the figure), the second column represents the set of assumptions implemented in the model. The third column represents the log-likelihood of the model (see Equation 5) at the best-fitting parameter values, and the fourth column represents the model's AIC value (see Equation 6). Finally, the last column represents the probability that the model is the best among those included in the comparison set (i.e., the AIC weight, see Equation 7). In these tables, VAR1 means equal variance, DS(Awr) means decisional separability for awareness, DS(Exp) means decisional separability for emotional expression, DS means decisional separability for awareness and emotional expression, PS(Awr) means perceptual separability for awareness, and "full" represents the unconstrained model without any of those assumptions. Across two experiments and two tasks, model number 7, which assumes equal variances and decisional separability for awareness, is the one that represents the data the best.

\subsection{Model Penalizing Unaware Sensitivity}

Although the SvA curve by itself should indicate whether discrimination sensitivity is above chance without awareness, we wanted to directly evaluate to what extent a model without unaware sensitivity would be able to account for the data. To do this, we fitted a full GRT-wIND model (see leftmost model in Figure 3) with a single constraint: it assumed chance-level discrimination sensitivity at the optimal bound for awareness. Unfortunately, this constraint cannot be imposed simply by fixing parameter values in the model, as the SvA curve is a complex function of the model parameters (see Equations 10 and 11), and therefore we could not fit this model using the same procedures that we used for all other models (i.e., maximizing the log-likelihood and constraining parameters to fixed values). Instead, this model was fitted by maximizing a penalized likelihood function $L L_{p}=L L-\alpha c$, where $c$ is a penalty term equal to the absolute value of conditional $d^{\prime}$ at $R L N A=1$, and $\alpha$ was set to 50. This allowed to maximize the likelihood of the model while penalizing any solutions yielding unaware sensitivity (i.e., conditional $d^{\prime}$ higher than zero at the bound separating the regions of high and low likelihood of awareness).

Unfortunately, it was very difficult to fit the parameters of this model without a failure of the fitting procedure, which we think was due to the fact that very few parameter settings allow the model to describe the data while adhering to the constraints imposed by the penalty term. For this reason, we used the full GRT-wIND model to ensure model flexibility; that is, to give the model the best ability to describe the data despite the constraints placed by the penalty term. In addition, we only performed the analysis using data from the discrimination task, as the more complex GRT model for the meta-cognitive task is likely to further complicate the optimization problem.

The penalized model was fit to the data 100 times, and each time the optimization procedure was started from a different random set of parameters. The best-fitting model of the set was retained, and we made sure that the SvA produced by the model showed a conditional $d^{\prime} \approx 0$ at $R L N A=1$.

The best-fitting penalized model is difficult to directly compare with all other models in the set (see Figure 3 in main manuscript), because the penalty term is not straightforwardly related to the parameters of the model. That is, although the penalty term places constraints on the values of 10 parameters involved in the final solution (by constraining conditional $d^{\prime}$, see Equation 10), those parameters are still free to vary in many other ways. For this reason, we compared the penalized model with all the other models in the set in two ways. The liberal comparison considered the full number of free parameters in the penalized model. The conservative approach subtracted the 10 parameters involved in the computation of conditional $d^{\prime}$ from the total number of parameters in the model. We believe that the liberal approach is fairer, as it reflects the 
true number of parameters that are free to vary in the penalized model, but the true ranking of the model is likely to land between the two extremes.

The results of the liberal comparison are presented in Tables S1 and S3, with the best-fitting penalized model highlighted in italics. In this liberal comparison, the model was ranked the worst in the analysis of data from Experiment 1 (Table S1), and 9th in the analysis of data from Experiment 2. Using the conservative approach did not change the conclusions: the model was ranked 13 th in the analysis of Experiment 1 $(\mathrm{AIC}=7768.419, \mathrm{AIC}$ weight $=0.000)$ and $3 \mathrm{rd}$ in the analysis of Experiment $2(\mathrm{AIC}=6037.718, \mathrm{AIC}$ weight $=0.003)$. In sum, using both a liberal and a conservative version of AIC to evaluate the penalized model, it did not provide a better description of the data than the best-fitting model with unaware sensitivity, and the AIC weights indicated that the model was very unlikely to be closest to the true data-generating model.

\subsection{Results of Model Comparison and Selection}

\begin{tabular}{ccccc}
\hline Model Number & Assumptions & Log-likelihood & AIC & AIC weight \\
\hline 7 & VAR1, DS(Awr) & -3682.589 & 7686.409 & 0.800 \\
14 & VAR1, DS(Awr), PS(Awr) & -3687.348 & 7689.211 & 0.197 \\
12 & VAR1, DS & -3735.066 & 7697.331 & 0.003 \\
16 & VAR1, DS, PS(Awr) & -3735.317 & 7708.194 & 0.000 \\
4 & DS(Awr) & -3681.609 & 7712.657 & 0.000 \\
15 & DS, PS(Awr) & -3738.076 & 7713.712 & 0.000 \\
9 & DS & -3734.343 & 7716.962 & 0.000 \\
11 & DS(Awr), PS(Awr) & -3694.634 & 7724.327 & 0.000 \\
6 & VAR1, DS(Exp) & -3709.309 & 7739.848 & 0.000 \\
13 & VAR1, DS(Exp), PS(Awr) & -3717.244 & 7749.002 & 0.000 \\
3 & DS(Exp) & -3704.456 & 7758.352 & 0.000 \\
10 & DS(Exp), PS(Awr) & -3713.082 & 7761.223 & 0.000 \\
2 & VAR1 & -3677.816 & 7805.087 & 0.000 \\
8 & VAR1, PS(Awr) & -3683.111 & 7806.313 & 0.000 \\
5 & PS(Awr) & -3679.236 & 7827.310 & 0.000 \\
1 & full & -3679.911 & 7848.965 & 0.000 \\
$P$ & Penalized Unaware Sensitivity & -3686.082 & 7861.307 & 0.000 \\
\hline
\end{tabular}

Table S1: Summary of the GRT models fitted to the discrimination task in Experiment 1

\begin{tabular}{ccccc}
\hline Model Number & Assumptions & Log-likelihood & AIC & AIC weight \\
\hline 7 & VAR1, DS(Awr) & -2690.934 & 6016.535 & 1.000 \\
14 & VAR1, DS(Awr), PS(Awr) & -2713.664 & 6048.040 & 0.000 \\
4 & DS(Awr) & -2694.319 & 6083.227 & 0.000 \\
11 & DS(Awr), PS(Awr) & -2721.230 & 6106.232 & 0.000 \\
16 & VAR1, DS, PS(Awr) & -2954.175 & 6243.408 & 0.000 \\
8 & VAR1, PS(Awr) & -2676.326 & 6255.388 & 0.000 \\
9 & DS & -2965.128 & 6279.697 & 0.000 \\
2 & VAR1 & -2677.115 & 6279.943 & 0.000 \\
12 & VAR1, DS & -2994.824 & 6310.878 & 0.000 \\
15 & DS, PS(Awr) & -3001.153 & 6337.364 & 0.000 \\
13 & VAR1, DS(Exp), PS(Awr) & -2953.275 & 6346.64 & 0.000 \\
3 & DS(Exp) & -2931.506 & 6352.155 & 0.000 \\
6 & VAR1, DS(Exp) & -2952.441 & 6354.336 & 0.000 \\
5 & PS(Awr) & -2698.878 & 6371.978 & 0.000 \\
10 & DS(Exp), PS(Awr) & -2954.149 & 6377.135 & 0.000 \\
1 & full & -2680.174 & 6386.810 & 0.000 \\
\hline
\end{tabular}

Table S2: Summary of the GRT models fitted to the metacognitive confidence rating task in Experiment 1 


\begin{tabular}{ccccc}
\hline Model Number & Assumptions & Log-likelihood & AIC & AIC weight \\
\hline 7 & VAR1, DS(Awr) & -2877.669 & 6025.988 & 0.988 \\
14 & VAR1, DS(Awr), PS(Awr) & -2885.563 & 6034.841 & 0.012 \\
11 & DS(Awr), PS(Awr) & -2880.183 & 6045.404 & 0.000 \\
4 & DS(Awr) & -2877.566 & 6055.284 & 0.000 \\
8 & VAR1, PS(Awr) & -2853.399 & 6075.924 & 0.000 \\
2 & VAR1 & -2853.661 & 6086.179 & 0.000 \\
5 & PS(Awr) & -2854.395 & 6107.967 & 0.000 \\
1 & full & -2847.874 & 6116.475 & 0.000 \\
$P$ & Penalized Unaware Sensitivity & -2856.701 & 6265.499 & 0.000 \\
6 & VAR1, DS(Exp) & -2948.261 & 6167.172 & 0.000 \\
3 & DS(Exp) & -2940.316 & 6180.784 & 0.000 \\
10 & DS(Exp), PS(Awr) & -2955.822 & 6196.681 & 0.000 \\
13 & VAR1, DS(Exp), PS(Awr) & -2968.054 & 6201.822 & 0.000 \\
9 & DS & -3028.152 & 6269.957 & 0.000 \\
16 & VAR1, DS, PS(Awr) & -3038.053 & 6278.575 & 0.000 \\
12 & VAR1, DS & -3047.846 & 6287.441 & 0.000 \\
15 & DS, PS(Awr) & -3045.869 & 6294.205 & 0.000 \\
& & & 0.000 \\
\hline
\end{tabular}

Table S3: Summary of the GRT models fitted to the discrimination task in Experiment 2

\begin{tabular}{ccccc}
\hline Model Number & Assumptions & Log-likelihood & AIC & AIC weight \\
\hline 7 & VAR1, DS(Awr) & -2661.641 & 5853.703 & 0.999 \\
14 & VAR1, DS(Awr), PS(Awr) & -2675.800 & 5873.394 & 0.001 \\
11 & DS(Awr), PS(Awr) & -2689.881 & 5947.035 & 0.000 \\
4 & DS(Awr) & -2678.037 & 5956.527 & 0.000 \\
2 & VAR1 & -2658.51 & 6104.111 & 0.000 \\
8 & VAR1, PS(Awr) & -2676.981 & 6116.628 & 0.000 \\
5 & PS(Awr) & -2666.808 & 6173.044 & 0.000 \\
1 & full & -2663.112 & 6223.224 & 0.000 \\
9 & DS & -3048.16 & 6396.473 & 0.000 \\
3 & DS(Exp) & -3049.312 & 6519.352 & 0.000 \\
6 & VAR1, DS(Exp) & -3080.202 & 6539.261 & 0.000 \\
15 & DS, PS(Awr) & -3135.652 & 6556.342 & 0.000 \\
16 & VAR1, DS, PS(Awr) & -3156.43 & 6597.897 & 0.000 \\
10 & DS(Exp), PS(Awr) & -3105.197 & 6609.571 & 0.000 \\
12 & VAR1, DS & -3216.949 & 6704.548 & 0.000 \\
13 & VAR1, DS(Exp), PS(Awr) & -3233.279 & 6835.686 & 0.000 \\
\hline
\end{tabular}

Table S4: Summary of the GRT models fitted to the metacognitive confidence rating task in Experiment 2 


\section{Estimated GRT-wIND models}

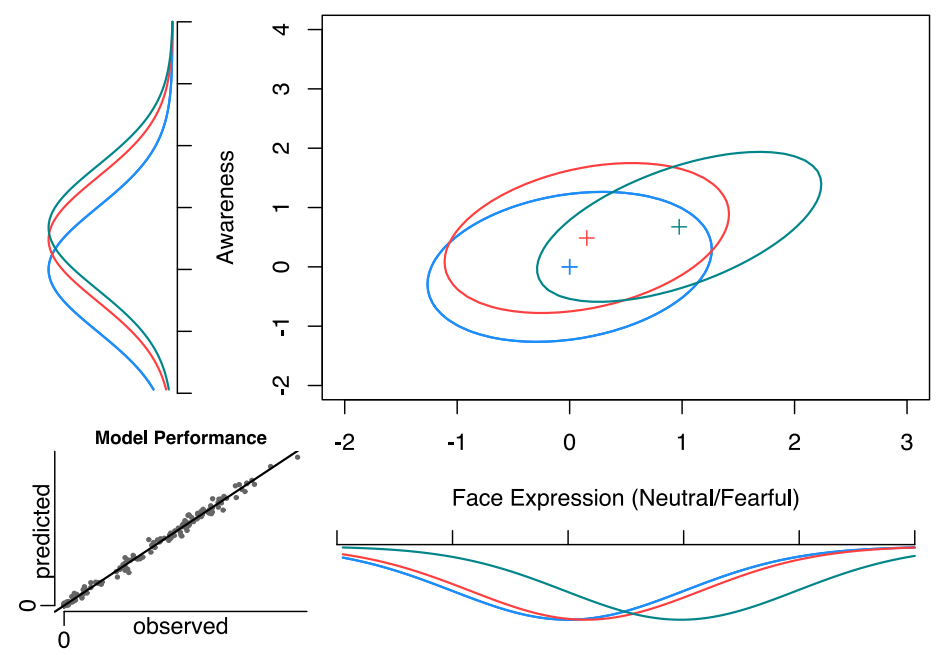

Figure S1: Best-fitting model from the analysis of the discrimination task in Experiment 1.

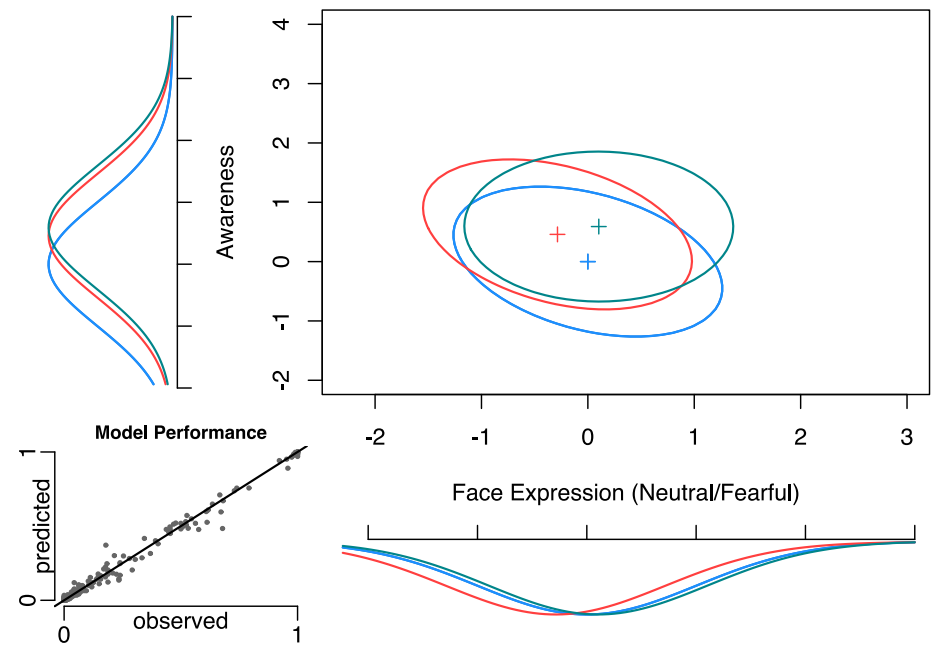

Figure S2: Best-fitting model from the analysis of confidence ratings (meta- $d$ ') in Experiment 1. 


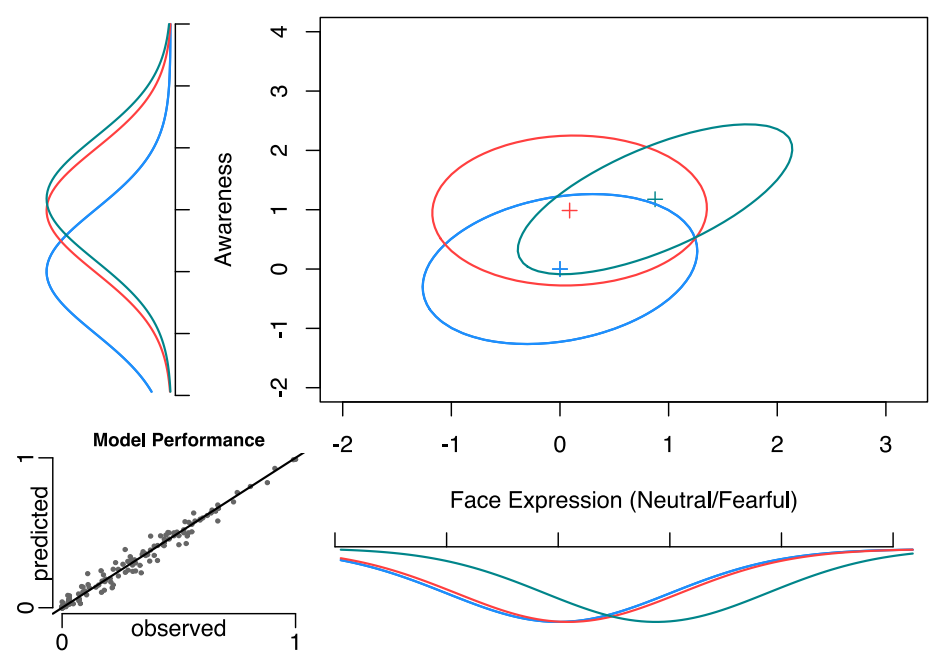

Figure S3: Best-fitting model from the analysis of the discrimination task in Experiment 2.

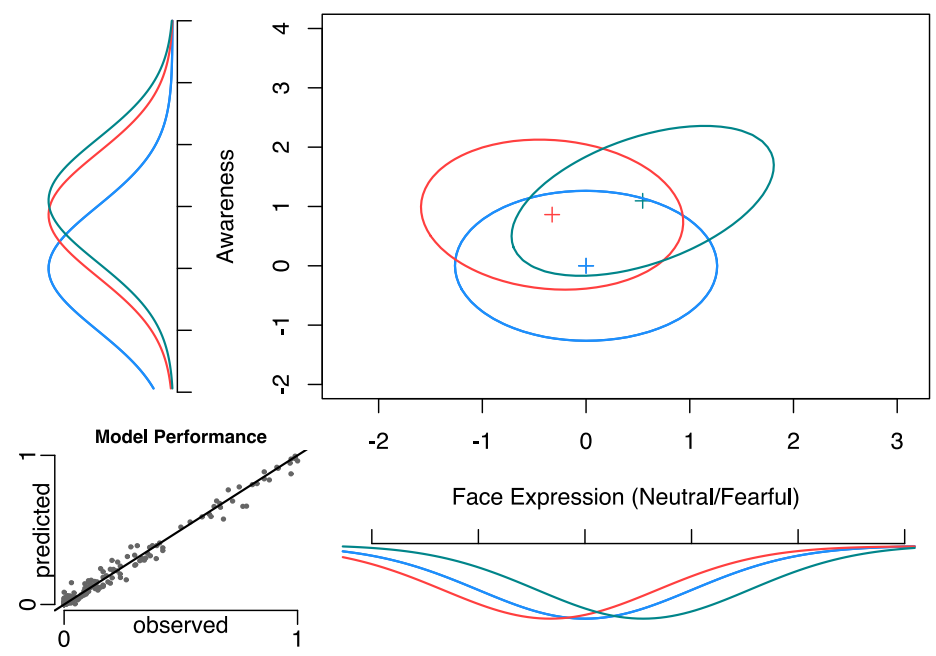

Figure S4: Best-fitting model from the analysis of confidence ratings (meta- $d$ ') in Experiment 2. 


\section{Individual SvA Curves}
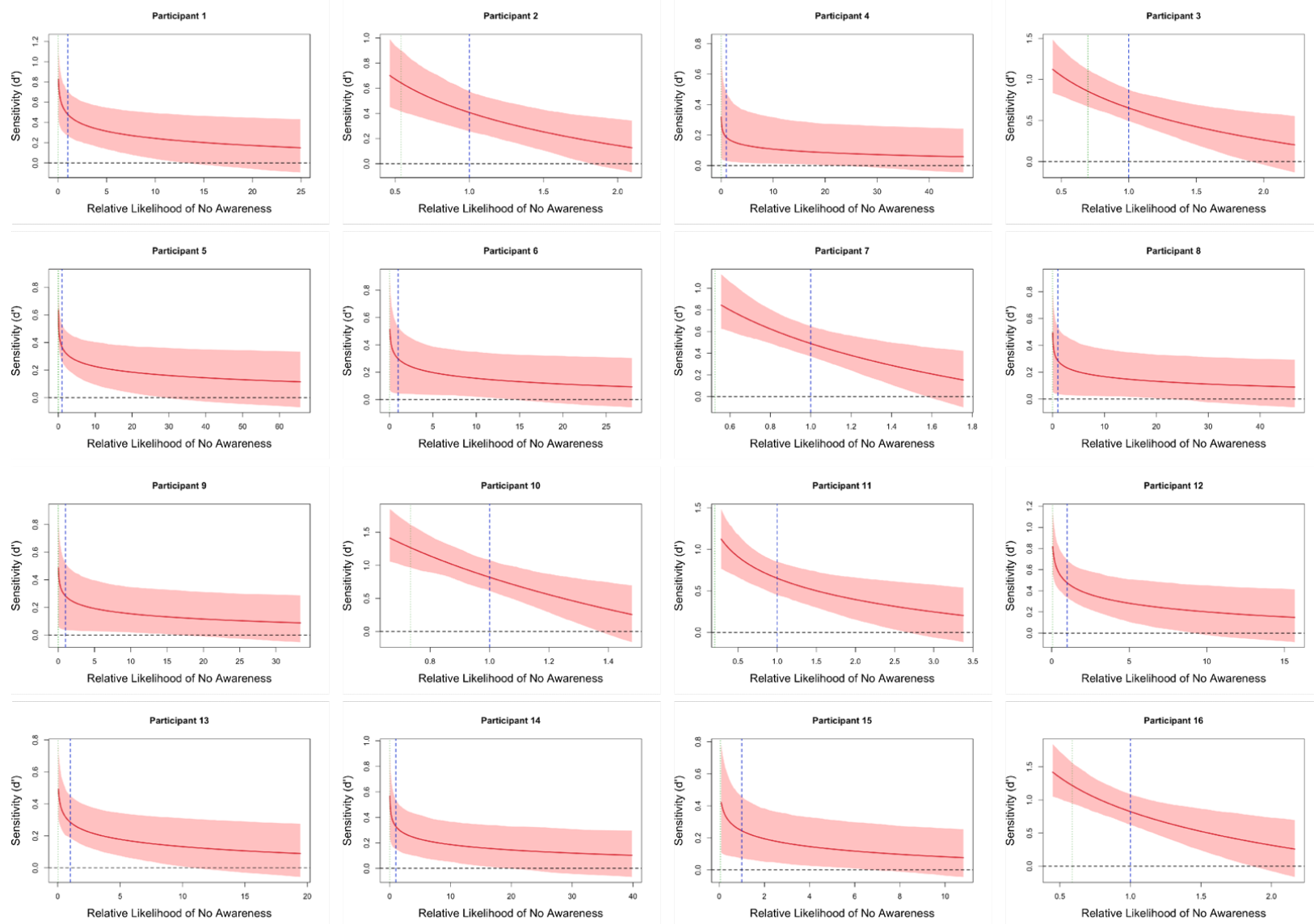

Figure S5: Individual SvA curves from the analysis of the discrimination task in Experiment 1 

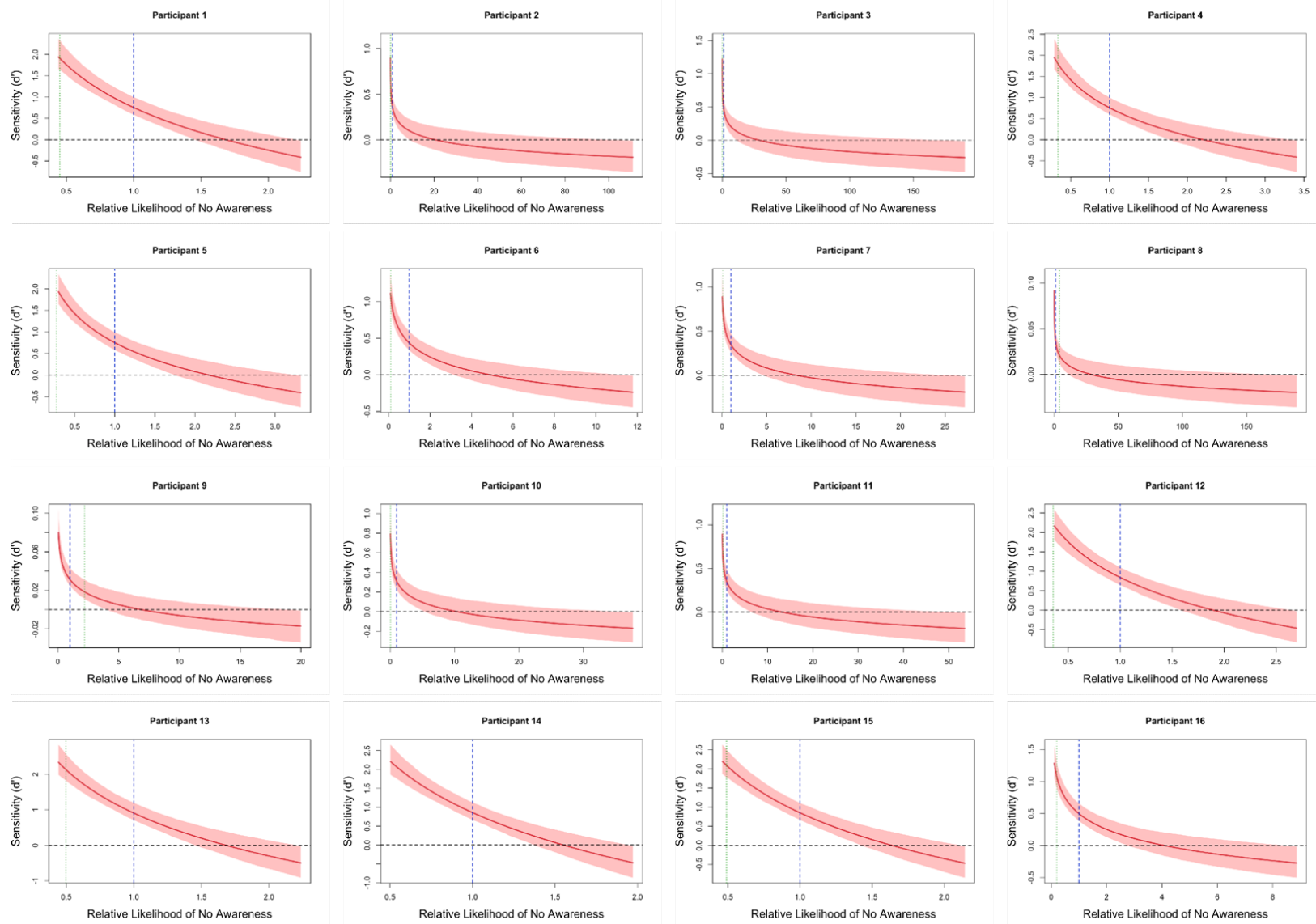

Figure S6: Individual SvA curves from the analysis of confidence ratings (meta- $d$ ') in Experiment 1 

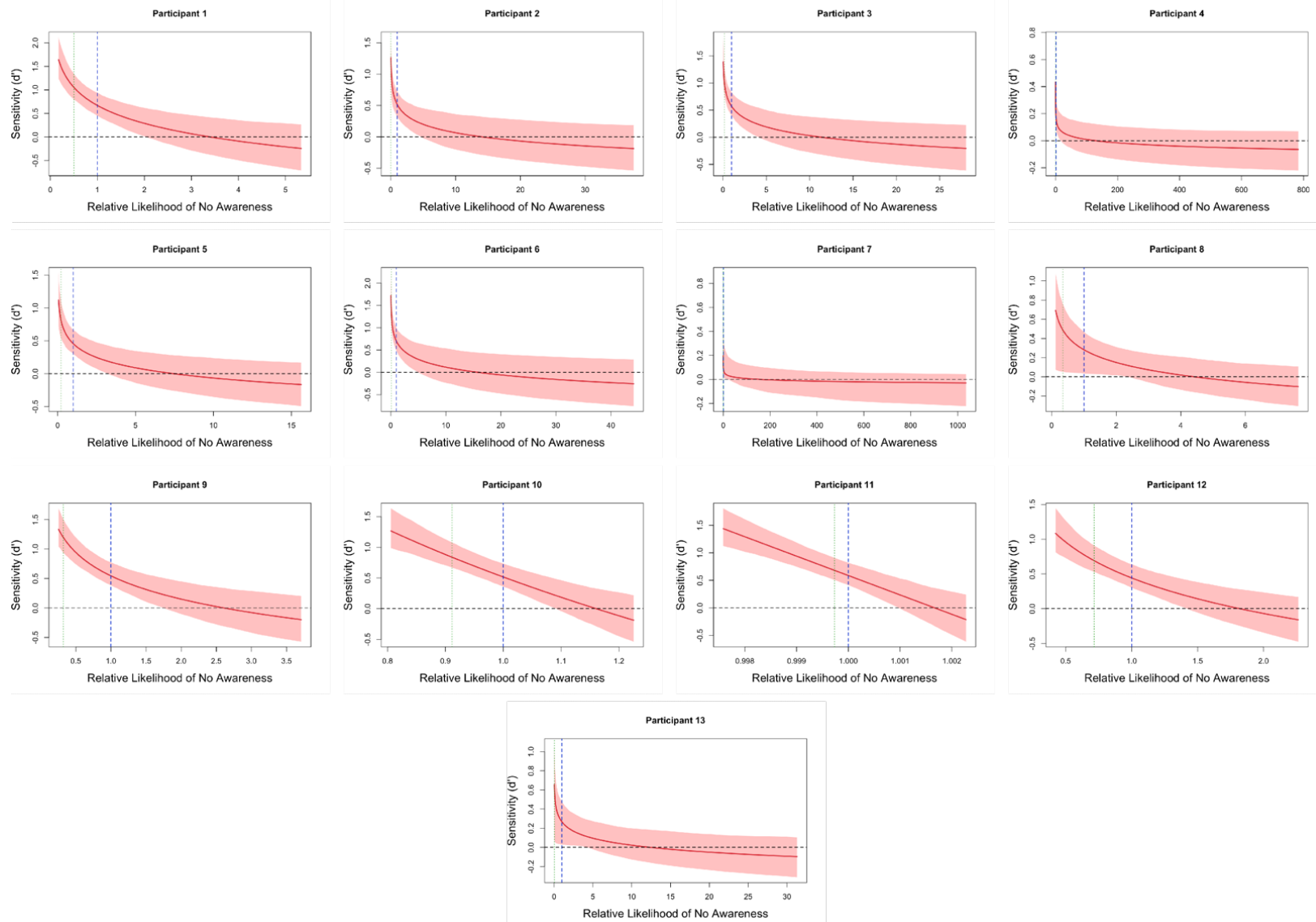

Figure S7: Individual SvA curves from the analysis of the discrimination task in Experiment 2 

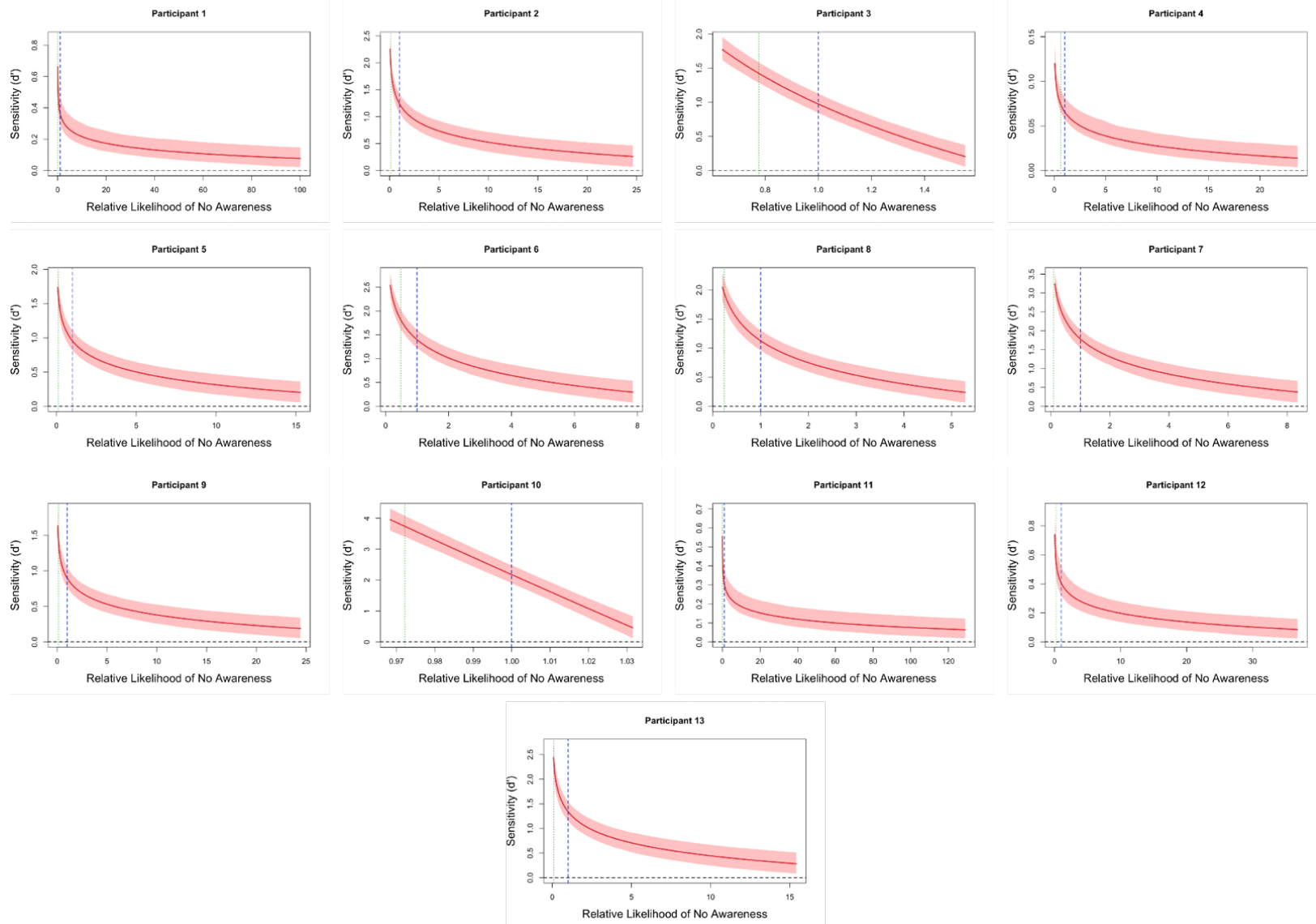

Figure S8: Individual SvA curves from the analysis of confidence ratings (meta- $d$ ') in Experiment 2

\section{References}

Ashby, F. G. \& Soto, F. A. (2015). Multidimensional signal detection theory. In J. Busemeyer, J. T. Townsend, Z. J. Wang, \& A. Eidels (Eds.), Oxford Handbook of Computational and Mathematical Psychology (pp. 13-34). New York, NY: Oxford University Press.

Carpenter, J. \& Bithell, J. (2000). Bootstrap confidence intervals: when, which, what? A practical guide for medical statisticians. Statistics in Medicine, 19(9), 1141-1164.

Good, P. I. (2006). Resampling methods: a practical guide to data analysis. Boston, MA: Birkhäuser.

Silbert, N. H. \& Thomas, R. D. (2017). Identifiability and testability in GRT with individual differences. Journal of Mathematical Psychology, 77, 187-196.

Soto, F. A., Vucovich, L., Musgrave, R., \& Ashby, F. G. (2015). General recognition theory with individual differences: A new method for examining perceptual and decisional interactions with an application to face perception. Psychonomic Bulletin \& Review, 22(1), 88-111.

Soto, F. A., Zheng, E., Fonseca, J., \& Ashby, F. G. (2017). Testing separability and independence of perceptual dimensions with general recognition theory: a tutorial and new R package (grtools). Frontiers in Psychology, 8, 696. 